Tôhoku Math. Journ.

39 (1987), 115-144.

\title{
STRONG AND CLASSICAL SOLUTIONS OF THE HOPF EQUATION -AN EXAMPLE OF FUNCTIONAL DERIVATIVE EQUATION OF SECOND ORDER
}

\author{
Atsushi InOUE
}

(Received March 5, 1986)

\begin{abstract}
In this note, we construct, strong and classical solutions of the Hopf equation, a statistical version of the Navier-Stokes equation on a compact Riemannian manifold with or without boundary. Our points are to regard the Hopf equation as a given Functional Derivative Equation (F.D.E. for short) of second order, to derive the Navier-Stokes equation as the characteristic equation of it and to give an exact meaning to the 'trace' of the second order functional derivatives which appear in the Hopf equation. To construct a solution of the Hopf-Foias equation with the energy inequality of strong form, we apply Foiaş's argument with slight modifications instead of using Prokhorov's compactness argument.
\end{abstract}

1. Introduction. Let $(M, g)$ be a compact Riemannian manifold of dimension $d$ with or without boundary $\partial M$. We denote by $\stackrel{\circ}{\boldsymbol{X}}_{\sigma}(M)$ and $\AA_{o}^{1}(M)$, the space of all solenoidal vector fields on $M$ which vanish near the boundary and that of all divergence free 1-forms on $M$ which vanish near the boundary, respectively. $\widetilde{\boldsymbol{H}}$ (resp. $\boldsymbol{H}$ ) stands for the completion of the space $\stackrel{\circ}{\Lambda}_{o}^{1}(M)$ (resp. $\left.\stackrel{\boldsymbol{X}}{\sigma}_{\sigma}(M)\right)$ with respect to $\widetilde{\boldsymbol{L}}^{2}$-norm (resp. $\boldsymbol{L}^{2}$-norm).

The aim of this paper is to solve the following problem.

(I) Find a real functional $W(t, \eta)$ on $[0, \infty) \times \widetilde{\boldsymbol{H}}$, satisfying

$$
\begin{aligned}
\frac{\partial}{\partial t} W(t, \eta)= & \int_{M}\left[-i\left\{\frac{\partial}{\partial x^{k}} \eta_{j}(x)-\Gamma_{j_{k}}^{l}(x) \eta_{l}(x)\right\} \frac{\delta^{2} W(t, \eta)}{\delta \eta_{j}(x) \delta \eta_{k}(x)}\right. \\
& \left.+\nu(\Delta \eta)_{j}(x) \frac{\delta W(t, \eta)}{\delta \eta_{j}(x)}+i \eta_{j}(x) f^{j}(x, t) W(t, \eta)\right] d_{g} x, \\
\frac{1}{\sqrt{g(x)}} & \frac{\partial}{\partial x^{j}}\left\{\sqrt{g(x)} \frac{\delta W(t, \eta)}{\delta \eta_{j}(x)}\right\}=0,
\end{aligned}
$$

for $\eta=\eta(x)=\eta_{j}(x) d x^{j} \in \AA_{o}^{1}(M)$ and $t \in(0, \infty)$, and

$$
\begin{gathered}
W(t, 0)=1, \\
W(0, \eta)=W_{0}(\eta) .
\end{gathered}
$$

Here $f(x, t)=f^{j}(x, t) \partial / \partial x^{j} \in \dot{X}_{o}(M)$ for each $t$ and $W_{0}(\eta)$ is a given positive definite functional on $\tilde{\boldsymbol{H}}$ satisfying 


$$
W_{0}(0)=1 \text { and } \frac{1}{\sqrt{g(x)}} \frac{\partial}{\partial x^{j}}\left\{\sqrt{g(x)} \frac{\delta W_{0}(\eta)}{\delta \eta_{j}(x)}\right\}=0 .
$$

Here and in what follows, we use Einstein's covention for contracting indices as well as the terminology and symbols in Riemannian geometry and functional analysis which will be explained in $\S 2$. There one also finds the definition of functional derivatives $\delta W(t, \eta) / \delta \eta_{j}(x)$ and $\delta^{2} W(t, \eta) / \delta \eta_{j}(x) \delta \eta_{k}(y)$.

In order to construct a solution of Problem (I), we consider the following problem.

(II) Find a family $\{\mu(t, \cdot)\}_{0<t<\infty}$ of Borel measures on $\boldsymbol{H}$ satisfying

$$
\begin{aligned}
& -\int_{0}^{\infty} \int_{H} \frac{\partial \Phi(t, u)}{\partial t} d \mu(t, u) d t-\int_{H} \Phi(0, u) d \mu_{0}(u) \\
& =\int_{0}^{\infty} \int_{H} \int_{M}\left[-u^{k}(x) u^{j}(x) \frac{\partial}{\partial x^{k}} \frac{\delta \Phi(t, u)}{\delta u^{j}(x)}+\Gamma_{k l}^{j}(x) u^{k}(x) u^{l}(x) \frac{\delta \Phi(t, u)}{\delta u^{j}(x)}\right. \\
& \left.\quad+\nu \nabla_{k} u^{j}(x) \cdot \nabla^{k} \frac{\delta \Phi(t, u)}{\delta u^{j}(x)}-f^{j}(x, t) \frac{\delta \Phi(t, u)}{\delta u^{j}(x)}\right] d_{g} x d \mu(t, u) d t
\end{aligned}
$$

for suitable test functionals $\Phi(t, u)$ which will be introduced in $\S 2$. Here, the given data are a measure $\mu_{0}$ and an exterior body force $f(t)$.

Our results are as follows, although we restate Theorem A more precisely as Theorem $\mathrm{A}^{\prime}$ later in $\S 5$ :

THEOREM A. For any Borel measure $\mu_{0}$ on $\boldsymbol{H}$ satisfying

$$
\int_{H}\left(1+|u|^{2}\right) d \mu_{0}(u)<\infty,
$$

and any $f(\cdot) \in L^{2}\left(0, \infty ; V^{-1}\right)$, there exists a solution $\{\mu(t, \cdot)\}_{0<t<\infty}$ of Problem (II).

Moreover, it satisfies the following energy inequality of strong form.

$$
\begin{aligned}
& \frac{1}{2} \int_{H} \psi\left(|u|^{2}\right) d \mu(t, u)+\nu \int_{0}^{t}\left[\int_{H} \psi^{\prime}\left(|u|^{2}\right)\|u\|^{2} d \mu(\tau, u)\right] d \tau \\
& \quad \leqq \frac{1}{2} \int_{H} \psi\left(|u|^{2}\right) d \mu_{0}(u)+\int_{0}^{t}\left[\int_{H} \psi^{\prime}\left(|u|^{2}\right)(f(\tau), u) d \mu(\tau, u)\right] d \tau
\end{aligned}
$$

for $0<t<\infty$ and $\psi \in C^{1}[0, \infty)$ satisfying

$$
0 \leqq \psi^{\prime}(t) \leqq \sup _{s \in[0, \infty)} \psi^{\prime}(s)<\infty .
$$

THEOREM B. Suppose a positive definite functional $W_{0}(\cdot)$ on $\tilde{\boldsymbol{H}}$ satisfy

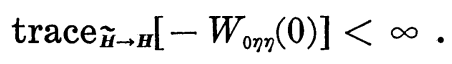

For any $f(\cdot) \in L^{2}\left(0, \infty ; V^{-1}\right)$, there exists a strong solution $W(t, \eta)$ of 
Problem (I).

THEOREM C. Let $\partial M=\varnothing$ and let $l$ be the largest integer not exceeding $(d / 2)+1$. Suppose a positive definite functional $W_{0}(\cdot)$ on $\tilde{\boldsymbol{H}}$ is of $\tilde{\boldsymbol{V}}^{-l}$ exponential type and satisfies

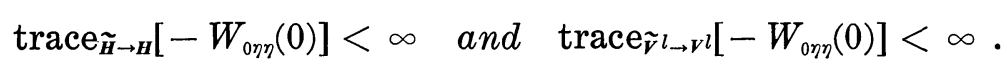

For any $f(\cdot)$ given in $L_{10 \mathrm{c}}^{1}\left(0, \infty ; V^{l}\right)$, there exists a classical solution $W(t, \eta)$ of Problem (I) on $\left[0, T^{*}\right)$ where $T^{*}<\infty$ is determined by $W_{0}$ and $f$ independently of $\nu$.

BRIEF HISTORY. The Hopf equation itself was introduced by Hopf [10], but he solved it only for a special case. (See, Hopf and Titt [11].)

Foiaș [5], [6] solved it in the form of Theorem A above on $M \times(0, T)$ when $M$ is a bounded domain in $\boldsymbol{R}^{d}, d \leqq 4$, and $T$ is an arbitrary but fixed number; It is remarkable that Gelfand [7] at I.C.M. in 1954 stressed the importance of F.D.E., in another word, analysis on functional spaces.

Since the work of Foiaș, there have appeared many papers statistically studying certain non-linear differential equations. For example, a statistical study of the non-linear Klein-Gordon equation was done by Vishik and Komech [22]. Especially, Ladyzhenskaya and Vershik [18] considered the following partial differential equation of infinitely many independent variables:

$$
\begin{aligned}
\frac{\partial}{\partial t} F(\theta, t)=-\nu \sum_{m=1}^{\infty} \lambda_{m} \theta_{m} \frac{\partial}{\partial \theta_{m}} F(\theta, t)-i \sum_{j, k, m=1}^{\infty} a^{j k, m} \theta_{m} & \frac{\partial^{2}}{\partial \theta_{j} \partial \theta_{k}} F(\theta, t) \\
& +i \sum_{m=1}^{\infty} \hat{f}_{m} \theta_{m} F(\theta, t) .
\end{aligned}
$$

It corresponds to Problem (II) for a bounded domain $M$ in $\boldsymbol{R}^{3}$. Their construction is based on the existence of weak solutions of the NavierStokes equation, and is different from that of Foias [5]. It seems desirable, however, to solve Problem (I) or (II) without the knowledge of the Navier-Stokes equation though the equations in Problems (I) and (II) are actually derived from it.

Vishik, Komech and Fursikov [23] considered also Problem (II), using Prokhorov's compactness argument.

On the other hand, Arsen'ev [1], [2] constructed another type of solution for Problem (II), called a turbulence measure for the Navier-Stokes equation, which seems to be a candidate for a weak solution.

In any case, there exists few papers dealing with the existence of the solution of F.D.E., except Donskar and Lions [3], Inoue, [12], [14], Levy [19], etc. 
REMaRKs. (1) The equation (I.1) is called the Hopf equation and that of (II) will be called the Hopf-Foiaș equation.

(2) Our main object is to solve a given F.D.E. like (I), and is not to repeat a statistical study of the Navier-Stokes equation, although we can extend a part of the results in Foias [5], [6] to the case where $T=\infty$ and $M$ is an arbitrary compact Riemannian manifold with or without boundary. Especially there is no restriction on the dimension $d$ of $M$. In Theorems $\mathrm{A}$ and $\mathrm{B}, M$ is rather arbitrary, but in Theorem $\mathrm{C}$, we must restrict our attention to the case where $\partial M=\varnothing$.

Terminologies used without definitions, for example, the definitions of strong and classical solutions, will be explained in $\S 2$. In $\S 3$, we derive Problem (II) from Problem (I) formally, that is, we show that the Navier-Stokes equation appears as the characteristic equation of the Hopf equation, by assuming some Ansatz's. Some functional spaces and Foiaș's compactness argument for a certain family of Borel measures are given in $\S 4$. We restate Theorem $A$ and prove it in $\S 5$. In $\S 6$, we give a strict meaning to the 'trace' of the second order functional derivatives in Problem (I). That is,

$$
\frac{\delta^{2} W(t, \eta)}{\delta \eta_{j}(x) \delta \eta_{k}(x)} \frac{\partial}{\partial x^{j}} \otimes \frac{\partial}{\partial x^{k}}
$$

makes sense as a distributional element of $\mathrm{ST}_{2}(M)$ in fairly general situations. This is a merit in considering the Navier-Stokes equation on a Riemannian manifold, and one of the motivations of writing this paper. There seem to exist no papers giving the meaning to the trace of higher order functional derivatives appearing formally in F.D.E. In $\S 7$, we give the proof of Theorem B. Foiaș [5] called a solution of (II) a statistical solution of the Navier-Stokes equation. We give the proof of Theorem $\mathrm{C}$ in $\S 8$. In $\S 9$, we mention some open problems.

A part of our results was announced in Inoue [13].

2. Preliminaries. (A) For later use, we recall some notation and symbols in Riemannian geometry.

Let $\left(g_{i j}(x)\right)$ be a positive definite, symmetric matrix on $M$ such that each element $g_{i j}(x)$ is smooth in $x$. The Riemannian metric on $M$ is formally denoted by $d g^{2}=g_{i j} d x^{i} d x^{j} . \quad g^{k l}(x)$ stands for the $(k, l)$-entry of the inverse of the matrix $\left(g_{i j}(x)\right)$. We put $g(x)=\operatorname{det}\left(g_{i j}(x)\right)$ and $d_{g} x=$ $\sqrt{g(x)} d x^{1} \wedge \cdots \wedge d x^{d}$ represents a volume element on $M$. (We abbreviate the argument $x$ if there occurs no confusion.)

Let us introduce some function spaces on $M$. 
$C^{\infty}(M)$ (resp. $\left.C_{0}^{\infty}(M)\right)$ denotes the space of smooth functions (resp. those with compact support, i.e., vanishing near the boundary) on $M$.

$\boldsymbol{X}(M)$ (resp. $\stackrel{\circ}{\boldsymbol{X}}(M)$, resp. $\stackrel{\circ}{\boldsymbol{X}}_{\sigma}(M)$ ) denotes the space of smooth (resp. with compact support, resp. with compact support and solenoidal) vector fields on $M$. We define $\boldsymbol{X}(\bar{M})$ and $\boldsymbol{X}_{o}(\bar{M})$ analogously. Here $\bar{M}=$ $M \cup \partial M$.

$\Lambda^{l}(M)$ denotes the space of smooth $l$-forms on $M$.

The space of symmetric tensor fields with $k$ contravariant (resp. covariant) indices is denoted by $\mathrm{ST}_{k}(M)$ (resp. $\left.\mathrm{ST}^{k}(M)\right)$. For example, $w \in \operatorname{ST}_{2}(M)$ means that it is expressed locally as

$$
w=w^{i j} \frac{\partial}{\partial x^{i}} \otimes \frac{\partial}{\partial x^{j}}
$$

with some functions $w^{i j}=w^{i j}(x)$ on $M$, symmetric in $i, j$. Analogously, $\zeta \in \mathrm{ST}^{2}(M)$ stands for

$$
\zeta=\zeta_{i j} d x^{i} \otimes d x^{j}
$$

with some functions $\zeta_{i j}=\zeta_{i j}(x)$ locally on $M$, symmetric in $i, j$.

Now, we put

$$
\begin{aligned}
& \Gamma_{i j}^{l}=\frac{1}{2} g^{l k}\left\{\frac{\partial}{\partial x^{i}} g_{k j}+\frac{\partial}{\partial x^{j}} g_{k i}-\frac{\partial}{\partial x^{k}} g_{i j}\right\}, \\
& R_{i j k}^{l}=\frac{\partial}{\partial x^{k}} \Gamma_{i j}^{l}-\frac{\partial}{\partial x^{j}} \Gamma_{i k}^{l}+\Gamma_{i j}^{m} \Gamma_{m k}^{l}-\Gamma_{i k}^{m} \Gamma_{m j}^{l}, \\
& R_{i j}=R_{i j m}^{m} \text { and } R_{j}^{i}=g^{i k} R_{k j} .
\end{aligned}
$$

For a 1 -form $\eta=\eta_{j} d x^{j}$, we define

$$
\begin{gathered}
\left(\nabla_{i} \eta\right)_{j}=\frac{\partial}{\partial x^{i}} \eta_{j}-\Gamma_{i j}^{l} \eta_{l}, \\
\left(\nabla^{i} \eta\right)_{j}=g^{i k}\left(\nabla_{k} \eta\right)_{j}=g^{i k}\left\{\frac{\partial}{\partial x^{k}} \eta_{j}-\Gamma_{j k}^{l} \eta_{l}\right\}, \\
\delta \eta=\frac{1}{\sqrt{g}} \frac{\partial}{\partial x^{i}}\left\{\sqrt{g} g^{i j} \eta_{j}\right\} \text { and }(\Delta \eta)_{j}=\nabla^{k} \nabla_{k} \eta_{j}+R_{j}^{k} \eta_{k} .
\end{gathered}
$$

Hereafter, we identify a 1 -form $\eta$ with a vector field $v$ by

$$
v^{i}=g^{i j} \eta_{j} \text { and } \eta_{i}=g_{i j} v^{j},
$$

which we also express, with abuse of notation, as

$$
v^{i}=(\tilde{\eta})^{i} \text { and } \eta_{i}=(\widetilde{v})_{i} .
$$

On the other hand, for a vector field $u=u^{j} \partial / \partial x^{j}$, we put 


$$
\begin{gathered}
\left(\nabla_{i} u\right)^{j}=\frac{\partial}{\partial x^{i}} u^{j}+\Gamma_{i k}^{j} u^{k}, \\
\left(\nabla^{i} u\right)^{j}=g^{i k}\left(\nabla_{k} u\right)^{j}=g^{i k}\left\{\frac{\partial}{\partial x^{k}} u^{j}+\Gamma_{k l}^{j} u^{l}\right\}, \\
\delta u=\frac{1}{\sqrt{g}} \frac{\partial}{\partial x^{i}}\left\{\sqrt{g} u^{i}\right\} \text { and }(\Delta u)^{j}=\nabla^{k} \nabla_{k} u^{j}-R_{k}^{j} u^{k}
\end{gathered}
$$

We also put

$$
\langle u, \eta\rangle=\int_{M} u_{i} \eta^{i} d_{g} x \text { for } u \in X(\bar{M}) \text { and } \eta \in \Lambda^{1}(\bar{M}) .
$$

For $u, v \in \boldsymbol{X}(\bar{M})$, we put

$$
(u, v)=\int_{M} g_{i j} u^{i} v^{j} d_{g} x=\langle u, \widetilde{v}\rangle=\langle\tilde{u}, v\rangle \text { and } \quad(u, u)=|u|^{2} .
$$

For $\eta, \xi \in \Lambda^{1}(\bar{M})$, we put

$$
(\eta, \xi)=\int_{M} g^{i j} \eta_{i} \xi_{j} d_{g} x=\langle\eta, \tilde{\xi}\rangle=\langle\tilde{\eta}, \xi\rangle \text { and }(\eta, \eta)=|\eta|^{2} .
$$

Moreover, we define

$$
((u, v))=\left(\nabla_{k} u, \nabla^{k} v\right)=\int_{M} g_{i j} \nabla_{k} u^{i} \nabla^{k} v^{j} d_{g} x \quad \text { and } \quad((u, u))=\|u\|^{2} .
$$

The completion of $\boldsymbol{X}(\bar{M})$ with respect to the norm $|\cdot|$ is denoted by $\boldsymbol{L}^{2}$. The closure of $\boldsymbol{X}_{\sigma}(\bar{M})$ in $\boldsymbol{L}^{2}$ is denoted by $\boldsymbol{H}$.

Let $s$ be a non-negative integer. In $X(\bar{M})$, we introduce the norm $\|\cdot\|_{s}$ defined by the Hilbert space structure

$$
((u, v))_{s}=\sum_{|\alpha| \leqq s}\left(\nabla^{(\alpha)} u, \nabla^{(\alpha)} v\right) \text { and }((u, u))_{s}=\|u\|_{s}^{2},
$$

where $u=u^{i} \partial / \partial x^{i} \in \boldsymbol{X}(\bar{M})$,

$$
\nabla^{(\alpha)}=\nabla_{1}^{\alpha_{1}} \cdot \nabla_{2}^{\alpha_{2}} \cdots \nabla_{d}^{\alpha_{d}}
$$

with $\alpha=\left(\alpha_{1}, \alpha_{2}, \cdots, \alpha_{d}\right)$ and $|\alpha|=\sum_{i=1}^{d} \alpha_{i}$.

$\boldsymbol{H}^{\boldsymbol{s}}$ (or $\boldsymbol{H}_{0}^{s}$ ) denotes the completion of $\boldsymbol{X}(\bar{M})$ (or $\stackrel{\circ}{\boldsymbol{X}}(M)$ ) with respect to the above norm $\|\cdot\|_{s}$. Clearly, we have

$$
((u, v))_{0}=(u, v), \quad((u, v))_{1}=(u, v)+((u, v)) \quad \text { and } \quad \boldsymbol{H}^{0}=\boldsymbol{L}^{2} .
$$

The closure of $\dot{X}_{\sigma}(M)$ in $\boldsymbol{H}^{\boldsymbol{s}}$ is denoted by $\boldsymbol{V}^{\boldsymbol{s}}$ and $\boldsymbol{V}^{1}$ is simply denoted by $\boldsymbol{V}$. By Poincaré's inequality, $\|\cdot\|$ is equivalent to $\|\cdot\|_{1}$ in our case. The dual space of $\boldsymbol{H}_{0}^{1}$ is denoted by $\boldsymbol{H}^{-1}$ and that of $\boldsymbol{V}^{\boldsymbol{s}}$ by $\boldsymbol{V}^{-\boldsymbol{s}}$.

$\boldsymbol{W}$ denotes the closure of $\dot{\boldsymbol{X}}_{\sigma}(M)$ in $\boldsymbol{H}^{1} \cap \boldsymbol{L}^{d}$ equipped with the norm

$$
\|u\|_{W}=\|u\|_{1}+|u|_{L^{d}},
$$


where $L^{d}$ stands for the space of vector fields whose components are $d$ summable on $M$ with respect to $d_{g} x$. In general, $\boldsymbol{W}$ is a subspace of $\boldsymbol{V}$ different from $V$, although $\boldsymbol{W}=\boldsymbol{V}$ for $d=2,3,4$. This seems to be the main reason why Foias restricts himself to the case $d \leqq 4$.

Analogously, we denote by $\widetilde{\boldsymbol{L}}^{2}, \widetilde{\boldsymbol{H}}, \widetilde{\boldsymbol{H}}^{s}, \widetilde{\boldsymbol{V}}, \widetilde{\boldsymbol{W}}$ and $\widetilde{\boldsymbol{V}}^{s}$, the completion of $\Lambda^{1}(\bar{M})$ or $\Lambda_{o}^{1}(\bar{M})$ or $\AA_{o}^{1}(M)$ with respect to the corresponding norms which are represented by the same symbols.

So, the dual space of $\boldsymbol{H}$ with respect to $\langle\cdot, \cdot\rangle$ or $(\cdot, \cdot)$ is $\widetilde{\boldsymbol{H}}$ or $\boldsymbol{H}$, respectively. Analogously, the dual space of $\boldsymbol{V}^{s}$ with respect to $\langle\cdot, \cdot\rangle$ or $(\cdot, \cdot)$ is $\tilde{V}^{-s}$ or $V^{-s}$, respectively.

(B) We next recall the definition of Fréchet derivatives.

DEFinition 2.1. (1) A real functional $\Phi(u)$ defined in a neighbourhood of a point $u_{0}$ of a Banach space $X$ with norm $|\cdot|$ is said to be Fréchet differentiable in $u$ at $u_{0}$, if there exists an element $\Phi_{u}\left(u_{0}\right) \in X^{\prime}$ such that

$$
\frac{1}{|v|}\left|\Phi\left(u_{0}+v\right)-\Phi\left(u_{0}\right)-\left\langle\Phi_{u}\left(u_{0}\right), v\right\rangle\right| \rightarrow 0
$$

as $|v| \rightarrow 0$, where $\langle$,$\rangle denotes the duality pairing between X$ and $X^{\prime}$.

(2) If the above holds for all $v$ belonging to a subspace $Y \subset X$, we shall call $\Phi$ Fréchet $X$-differentiable at $u_{0}$ in the direction of $Y$. In both cases, $\Phi_{u}\left(u_{0}\right)$ is called the Fréchet differential of $\Phi$ in $u$ at $u_{0}$.

(3) For $X=Y \times Z$, we write

$$
\Phi_{y, z}\left(y_{0}, z_{0}\right)=\left(\Phi_{y}\left(y_{0}, z_{0}\right), \Phi_{z}\left(y_{0}, z_{0}\right)\right),
$$

where the components constitute the partial Fréchet differentials.

Definition 2.2. A Fréchet differentiable functional $\Phi$ is twice Fréchet differentiable at $u_{0}$ if $\Phi_{u}: X \rightarrow X^{\prime}$ is differentiable. That is, there exists an element $\Phi_{u u}\left(u_{0}\right) \in(X \otimes X)^{\prime}$ such that

$$
\frac{1}{|v|} \sup _{w \in X,|w| \leqq 1}\left|\left\langle\Phi_{u}\left(u_{0}+v\right), w\right\rangle-\left\langle\Phi_{u}\left(u_{0}\right), w\right\rangle-\left\langle\Phi_{u u}\left(u_{0}\right), v \otimes w\right\rangle\right| \rightarrow 0
$$

as $|v| \rightarrow 0$.

(C) Now, we define functional derivatives.

Let $E$ be a function space on $M$ containing $\left(C_{0}^{\infty}(M)\right)^{m}$ whose dual space, denoted by $E^{\prime}$, is contained in $\left(\mathscr{D}^{\prime}(M)\right)^{m}$ : We assume implicitly that $E$ has at least a structure of a locally convex topological vector space. Here $C_{0}^{\infty}(M)$ denotes the space of infinitely differentiable functions with compact support and $\mathscr{D}^{\prime}(M)$ denotes the space of distributions on $M$. 〈, > stands for the duality pairing between $E$ and $E^{\prime}$. (It represents 
also other duality pairings which will be explained for each instance, if necessary.)

First of all, we consider the case $m=1$.

Definition 2.3. Let $\Phi=\Phi(f)$ be a functional on $E$. If at $f \in E$, there exists $D \Phi(f) \in E^{\prime}$ such that

$$
\left.\frac{d}{d \varepsilon} \Phi(f+\varepsilon h)\right|_{\varepsilon=0}=\langle D \Phi(f), h\rangle,
$$

then $\Phi$ is said to be differentiable (or more precisely, Geteaux-differentiable) at $f$ (in the direction of $h$ ). We represent formally the right hand side of (2.1) as

$$
\langle D \Phi(f), h\rangle=\int_{M} \frac{\delta \Phi(f)}{\delta f(x)} h(x) d_{g} x \quad \text { for } \quad h \in C_{0}^{\infty}(M) .
$$

$\delta \Phi(f) / \delta f(x)$, being a distribution on $M$, is sometimes called the functional derivative of $\Phi$ by $f$ at $x$ or of $\Phi$ at $f(x)$.

In physics literature, we find the following abbreviation for the above definition, even though $\delta(\cdot)$ is not contained in $E$.

$$
\frac{\delta \Phi(f)}{\delta f(x)}=\left.\frac{d}{d \varepsilon} \Phi(f(\cdot)+\varepsilon \delta(\cdot-x))\right|_{\varepsilon=0} .
$$

Definition 2.4. Let $\Phi$ be a differentiable functional on $E$. If $\left\langle D \Phi(f), h_{1}\right\rangle$ is differentiable as a functional of $f$ for each $h_{1} \in E$, then $\Phi$ is called twice differentiable at $f$ and its second derivative $D^{2} \Phi(f)$ is given by

$$
\left.\frac{d}{d \varepsilon}\left\langle D \Phi\left(f+\varepsilon h_{2}\right), h_{1}\right\rangle\right|_{\varepsilon=0}=\left\langle D^{2} \Phi(f), h_{1} \otimes h_{2}\right\rangle
$$

Here, $\langle$,$\rangle appearing on the right-hand side of (2.2) stands for the duality$ pairing between $E=E(M \times M)$ and $E^{\prime}=E^{\prime}(M \times M)$ and $\left(h_{1} \otimes h_{2}\right)(x, y)=$ $h_{1}(x) h_{2}(y), h_{1}, h_{2} \in C_{0}^{\infty}(M)$ and $x, y \in M$. Moreover, by the kernel theorem of Schwartz [9], we may express the right-hand side of (2.2) as

$$
\begin{aligned}
\left\langle D^{2} \Phi(f), h_{1} \otimes h_{2}\right\rangle & =\int_{M \times M} \frac{\delta^{2} \Phi(f)}{\delta f(x) \delta f(y)} h_{1}(x) h_{2}(y) d_{g} x d_{g} y \\
& =\int_{M \times M} \frac{\delta^{2} \Phi(f)}{\delta f(y) \delta f(x)} h_{1}(x) h_{2}(y) d_{g} x d_{g} y .
\end{aligned}
$$

Analogously, we define

$$
\left\langle D^{n} \Phi(f), h_{1} \otimes \cdots \otimes h_{n}\right\rangle=\int_{M \times \ldots \times M} \frac{\delta^{n} \Phi(f)}{\delta f\left(x_{1}\right) \cdots \delta f\left(x_{n}\right)} h_{1}\left(x_{1}\right) \cdots h_{n}\left(x_{n}\right) d_{g} x_{1} \cdots d_{g} x_{n} \text {. }
$$


If a functional $\Phi$ depends on many variables $\left(f_{1}, f_{2} \cdots f_{m}\right)$, then we may define partial functional derivative $\delta \Phi\left(f_{1}, f_{2} \cdots f_{m}\right) / \delta f_{j}(x)$ analogously. Higher order derivatives as well as higher order partial derivatives of a functional with many variables are defined analogously. (cf. Volterra [24].)

REMARK 2.5. Since $\delta^{2} \Phi(f) / \delta f(x) \delta f(y)$ is a distribution on $M \times M$, it generally does not make sense to put $x=y$. But if it is possible to regard something like $\left.\left(\delta^{2} \Phi(f) / \delta f(x) \delta f(y)\right)\right|_{x=y}$ as a distribution on $M$, we denote it by $\delta^{2} \Phi(f) / \delta f(x)^{2}$, and call it the trace of $\delta^{2} \Phi(f) / \delta f(x) \delta f(y)$. Analogously, we define $\delta^{n} \Phi(f) / \delta f(x)^{n}$ etc.

(D) Now, we proceed to define the notion of solutions of (I).

We introduce:

Definition 2.6. A real functional $\Phi(\cdot, \cdot)$ on $[0, \infty) \times \boldsymbol{H}$ is called an elementary test functional if it satisfies the following conditions.

(1) $\Phi(\cdot, \cdot)$ satisfies

$$
\Phi(t, u)=\Phi\left(t, P_{m} u\right) \quad \text { for } \quad(t, u) \in[0, \infty) \times \boldsymbol{H}
$$

for a certain $m \in \boldsymbol{N}$ depending on $\Phi$.

(2) $\Phi(\cdot, \cdot)$ is continuously Fréchet differentiable from $[0, \infty) \times \boldsymbol{H}$ to $\boldsymbol{R}$ and there exist constants $c_{j}, j=1,2,3$, depending on $\Phi$ such that for all $(t, u) \in[0, \infty) \times \boldsymbol{H}$, we have

$$
\left|\Phi_{u}(t, u)\right| \leqq c_{1} \text { and }\left|\Phi_{t}(t, u)\right| \leqq c_{2}+c_{3}|u| \text {. }
$$

Here, $\Phi_{u}(t, \cdot)$ is regarded as an element in $\widetilde{\boldsymbol{H}}$.

Definition 2.7. A real functional $\Phi(\cdot, \cdot)$ defined on $[0, \infty) \times V$ is called a test functional if it satisfies the following.

(1) $\Phi(\cdot, \cdot)$ is continuous on $[0, \infty) \times V$ and verifies $(2.3)$.

(2) $\Phi(\cdot, \cdot)$ is Fréchet $\boldsymbol{H}$-differentiable in the direction $\boldsymbol{V}$.

(3) $\Phi_{u}(\cdot, \cdot)$ is continuous from $[0, \infty) \times V$ to $\widetilde{\boldsymbol{V}}^{s}$ and is bounded. That is, there exists a constant $c_{4}$ depending on $\Phi$ such that

$$
\left\|\Phi_{u}(t, u)\right\|_{s} \leqq c_{4} \text { for all }(t, u) \in[0, \infty) \times \boldsymbol{V} .
$$

The set of all test functionals (resp. elementary test functionals) is denoted by TF (resp. ETF).

Now, we give:

Definition 2.8. A family of Borel measures on $\boldsymbol{H}$ is called a solution fo Problem (II) on $(0, \infty)$ if it satisfies (II) and the following conditions:

(H.1) $\int_{H}\left(1+|u|^{2}\right) d u(\cdot, u) \in L^{\infty}(0, \infty)$ 
(H.2) $\int_{H}\|u\|^{2} d \mu(\cdot, u) \in L^{1}(0, \infty)$.

(H.3) $\int_{H} \Phi(u) d \mu(t, u)$ is measurable in $t$ for any non-negative, weakly continuous functional $\Phi(\cdot)$ on $\boldsymbol{H}$.

Definition 2.9. A functional defined on $[0, T) \times \widetilde{\boldsymbol{H}},(T \leqq \infty)$ will be called a strong solution of Problem (I) on $(0, T)$ if there exists a set $\widetilde{D}$, dense in $\widetilde{\boldsymbol{V}}^{s}$, for some $s$, containing $\AA_{\sigma}^{1}(M)$ such that:

(1) For each $\eta \in \widetilde{D}, W(t, \eta)$ belongs to $L_{10 \mathrm{c}}^{1}[0, T)$ is continuous in $t$ at $t=0$ and is twice differentiable at $\eta \in \widetilde{D}$ for a.e.t.

(2) $\frac{\delta^{2} W(t, \eta)}{\delta \eta_{j}(x) \delta \eta_{k}(x)} \frac{\partial}{\partial x^{j}} \otimes \frac{\partial}{\partial x^{k}}$

exists for almost every $t$ on $(0, T)$ as a distributional element in $\operatorname{ST}_{2}(M)$ for $\eta \in \widetilde{D}$.

(3) $W(t, \eta)$ satisfies (I.1)-(I.4) as distributions for each $\eta \in \widetilde{D}$.

Definition 2.10. A functional defined on $[0, T) \times \widetilde{\boldsymbol{H}},(T \leqq \infty)$ will be called a classical solution of Problem (I) on $(0, T)$ if there exists a dense set $\widetilde{D}$ in $\widetilde{V}^{s}$, for some $s$, containing $\Lambda_{\sigma}^{1}(M)$ such that:

(1) $W(t, \eta)$ is absolutely continuous on $[0, T)$ for each $\eta \in \widetilde{D}$ and is twice differentiable; moreover, $\delta W(t, \eta) / \delta \eta_{j}(x)$ belongs to $L_{10 \mathrm{c}}^{1}(M)$ for each $j$.

(2) $\delta^{2} W(t, \eta) / \delta \eta_{j}(x) \delta \eta_{k}(x)$ exists for each $j, k$ and almost every $t$ on $[0, T)$ as an element of $L_{10 \mathrm{c}}^{1}(M)$ for $\eta \in \widetilde{D}$. Moreover,

$$
\frac{\delta^{2} W(t, \eta)}{\delta \eta_{j}(x) \delta \eta_{k}(x)} \frac{\partial}{\partial x^{j}} \otimes \frac{\partial}{\partial x^{k}}
$$

belongs to $\mathrm{ST}_{2}(M)$ as an element in $L_{1 \mathrm{oc}}^{1}(M)$.

(3) $W(t, \eta)$ satisfies (I.1)-(I.4) for almost every $t$ as functions for each $\eta \in \widetilde{D}$.

REMARK 2.11. There may be many other possibilities of the notion of solutions, for example, those which will be defined by respecting the uniqueness or the regularity of solutions.

Finally, we introduce:

Definition 2.12. A positive definite functional $W$ on $\tilde{\boldsymbol{H}}$ is said to be of $\widetilde{\boldsymbol{V}}^{-\boldsymbol{s}}$ exponential type for any $\eta \in \tilde{\boldsymbol{H}}$, if the function $s \rightarrow W(s \eta)$ defined on $\boldsymbol{R}$ can be extended analytically to an entire function $W(\zeta ; \eta)$ on the complex plane $\boldsymbol{C}$ satisfying

$$
|W(\zeta ; \eta)| \leqq c_{5} \cdot e^{c_{\theta}|\mathrm{Im} \zeta|\|\eta\| \mid-s} \quad \text { for all } \zeta \in \boldsymbol{C}, \eta \in \widetilde{\boldsymbol{H}},
$$


where $c_{5}$ and $c_{8}$ are constants depending on $\boldsymbol{W}$.

3. The derivation of Problem (II) from Problem (I). Multiplying both sides of (I.1) by a function $\rho(t) \in C_{0}^{\infty}[0, \infty)$ and integrating with respect to $t$, we get

$$
\begin{aligned}
& -\int_{0}^{\infty} \rho^{\prime}(t) W(t, \eta) d t-\rho(0) W(0, \eta) \\
& =\int_{0}^{\infty} \rho(t) \int_{M}\left[-i\left\{\frac{\partial}{\partial x^{k}} \eta_{j}(x)-\Gamma_{j k}^{l}(x) \eta_{l}(x)\right\} \frac{\delta^{2} W(t, \eta)}{\delta \eta_{j}(x) \delta \eta_{k}(x)}\right. \\
& \left.\quad+\nu(\Delta \eta)_{j}(x) \frac{\delta W(t, \eta)}{\delta \eta_{j}(x)}+i \eta_{j}(x) f^{j}(x, t) W(t, \eta)\right] d_{g} x d t .
\end{aligned}
$$

ANSATZ 1. There exists a family $\{\mu(t, \cdot)\}_{0<t<\infty}$ of Borel measures on the set $\mathscr{B}\left(\boldsymbol{L}^{2}\right)$ of Borel sets in $\boldsymbol{L}^{2}$ such that

$$
W(t, \eta)=\int_{L^{2}} e^{i\langle v, \eta\rangle} d \mu(t, v) .
$$

Then, (I.3) and (I.4) yield that

$$
\int_{L^{2}} d \mu(t, v)=1 \text { for } 0<t<\infty
$$

and

$$
W_{0}(\eta)=\int_{L^{2}} e^{i\langle v, \eta\rangle} d \mu_{0}(v) .
$$

Moreover, by (I.2), measures $\mu(t, \cdot)$ must be supported on $\boldsymbol{H}$. That is,

$$
\frac{1}{\sqrt{g(x)}} \frac{\partial}{\partial x^{j}}\left\{\sqrt{g(x)} v^{j}(x)\right\}=0
$$

for $\boldsymbol{v}(\cdot)=v^{j}(\cdot) \partial / \partial x^{j} \in \operatorname{supp} \mu(t, \cdot) \subset \boldsymbol{L}^{2}$

Analogously, if $W_{0}(\eta)$ satisfies the condition (I.5), then

$$
\text { supp } \mu_{0} \subset \boldsymbol{H} \text { and } \int_{L^{2}} d \mu_{0}(u)=1 \text {. }
$$

Substitute (3.2) into (3.1) and assume that functionals $\Phi(t, v ; \eta)=$ $\rho(t) e^{i\langle v, \eta\rangle}$ belong to the space of test functionals. Then by the definition of functional derivatives and (3.5), we get

$$
\begin{aligned}
& -i \rho(t) \int_{M}\left\{\frac{\partial}{\partial x^{k}} \eta_{j}(x)-\Gamma_{j k}^{l}(x) \eta_{l}(x)\right\} \frac{\delta^{2} W(t, \eta)}{\delta \eta_{j}(x) \delta \eta_{k}(x)} d_{g} x \\
& =-i \int_{H}\left[\int_{M}\left\{\frac{\partial}{\partial x^{j}} \eta_{i}(x)-\Gamma_{i j}^{l}(x) \eta_{l}(x)\right\} v^{i}(x) v^{j}(x) d_{g} x\right] \Phi(t, v ; \eta) d \mu(t, v) \\
& =-\int_{H} \int_{M}\left\{v^{k}(x) \frac{\partial}{\partial x^{k}} v^{j}(x)+\Gamma_{k l}^{j}(x) v^{k}(x) v^{l}(x)\right\} \frac{\delta \Phi(t, v ; \eta)}{\delta v^{j}(x)} d_{g} x d \mu(t, v) .
\end{aligned}
$$


Calculating analogously other terms on the right hand side of (3.1), we get (II.1) for $\Phi(t, v ; \eta)$. Here, we assume implicitly that $\delta^{2} W(t, \eta) / \delta \eta_{j}(x) \delta \eta_{k}(x)$ exists for $W(t, \eta)$ expressed by (3.2) and also that the change of the order of the integration with respect to $d_{g} x$ and $d \mu(t, u)$ is permissible.

Let us introduce another:

Ansatz 2. A family $\{\mu(t, \cdot)\}_{0<t<\infty}$ of Borel measures in (3.2) is induced from an operator $T_{t}$ in $\boldsymbol{L}^{2}$ for $0<t<\infty$, that is,

$$
\mu(t, \omega)=\mu_{0}\left(T_{t}^{-1} \omega\right) \text { for } 0<t<\infty \text { and } \omega \in \mathscr{B}\left(\boldsymbol{L}^{2}\right) .
$$

In other words,

$$
W(t, \eta)=\int_{L^{2}} e^{i\left\langle T t^{u, \eta\rangle}\right.} d \mu_{0}(u) .
$$

Combining this with (3.4) and (3.6), we get

$$
T_{0} u=u \text { in } \boldsymbol{H} \text {. }
$$

By (3.7), (I.2) and the definition of the functional deriative, we get

$$
\begin{aligned}
\left.\frac{d}{d \varepsilon} W(t, \eta+\varepsilon d \varphi)\right|_{\varepsilon=0} & =\int_{L^{2}}\left\langle T_{t} u, d \varphi\right\rangle e^{i\left\langle T_{t} u, \eta\right\rangle} d \mu_{0}(u) \\
& =\left\langle d \varphi,\left\{\frac{\delta W(t, \eta)}{\delta \eta_{j}}\right\}\right\rangle=\left\langle\varphi, \delta\left\{\frac{\delta W(t, \eta)}{\delta \eta_{j}}\right\}\right\rangle=0
\end{aligned}
$$

for any $\varphi \in C_{0}^{\infty}(M)$ and for any $\eta \in \boldsymbol{L}^{2}$. So, it seems natural to assume

$$
\left\langle T_{t} u, d \varphi\right\rangle=0 \text { for any } \varphi \in C_{0}^{\infty}(M) .
$$

That is, by (3.5) and (3.9),

$$
T_{t} u \in \boldsymbol{H} \text { for } u \in \boldsymbol{H} .
$$

Substituting (3.8) and (3.11) into (I.1), we have

$$
i \int_{L^{2}}\left\langle\left(T_{t} u\right)^{\prime}-\nu \Delta T_{t} u+\nabla_{T_{t} u} \cdot T_{t} u-f, \eta\right\rangle e^{i\left\langle T_{t} u, \eta\right\rangle} d \mu_{0}(u)=0 .
$$

Combining the above results, we have finally:

ANSATz. There exists a family $\left\{T_{t}\right\}$ of operators in $\boldsymbol{H}$ and a Borel measure $\mu_{0}$ such that a solution of (I) is expressed as

$$
W(t, \eta)=\int_{H} e^{i\left\langle T_{t} u, \eta\right\rangle} d \mu_{0}(u) .
$$

Then, by the Hodge decomposition, we get that $T_{t} u(=u(\cdot, t))$ is a solution of the following initial boundary value problem for the NavierStokes equation. 


$$
\begin{gathered}
\frac{\partial}{\partial t} u(x, t)-\nu \Delta u(x, t)+\nabla_{u(x, t)} \cdot u(x, t)+\nabla \pi(x, t)=f(x, t), \\
\delta u(x, t)=0 \\
\left.u(x, t)\right|_{\partial M}=0 \text { and } \\
u(x, 0)=u_{0}(x) .
\end{gathered}
$$

REmark. (1) Above Ansatz asserts that the Hopf equation has the Navier-Stokes equation as the characteristic equation. Note, however, that the uniqueness of the weak solution of the Navier-Stokes equation is not known. Hence the above operator $T_{t}^{-1}$ does not necessarily exist.

(2) The condition (1.2), which follows from the gauge condition (3.14), has a counterpart in Quantum Field Theory, called the WardTakahashi identity.

(3) Hopf [10] and Foiaș [5], derived (I) or (II) from the Navier-Stokes equation by an argument converse to ours above. It seems meaningful, however, to start with a given F.D.E. just as we treat the Schrödinger equation independently of the fact that it is derived from the classical mechanics. Unfortunately, there does not exist any mathematical foundation for treating F.D.E. directly, though it was possible to regard the Schrödinger equation as an example of P.D.E., when it appeared.

4. Some functional spaces and Foiaș's compactness argument for a certain family of Borel measures. Concerning the criterion for the weak compactness of measures, Prokhorov's theorem is now well-known. (See, Theorem 1, §1, Chap. VI in Gihman and Skorohod [9].) But we follow essentially the argument of Foias [5] and present here Foias's result in somewhat more abstract form. The detailed proof will not be presented here, because we can get them slightly modifying the arguments in [5].

Let $X, Y$ be Banach spaces such that $Y \subset X$ where the injection is dense, continuous and compact. We denote the norms of $X$ and $Y$ by $|\cdot|$ and $\|\cdot\|$, respectively. Moreover, we assume that there exists a family of operators $P_{m}$ in $X$ for $m=1,2, \cdots$ with the following properties.

(P.1) $\quad P_{m} \rightarrow I$ as $m \rightarrow \infty$ in $X$ and $P_{m} u \in D$ for all $u \in X$.

(P.2) For each $m$, if $u$ converges to $v$ weakly in $X$, then $P_{m} u$ converges to $P_{m} v$ in $Y$.

(P.3) $\left\|P_{k} u\right\| \leqq\left\|P_{k+1} u\right\| \leqq\|u\|$ for $u \in X$.

Here, $D$ is a dense subset in $Y$.

As functional spaces, we introduce the following:

Definition 4.1. (i) $C_{\alpha}$ for $\alpha \geqq 0$ denotes the space of all real continuous functionals $\Phi(\cdot)$ on $X$ such that 


$$
\|\Phi\|_{c_{\alpha}}=\sup _{u \in X} \frac{|\Phi(u)|}{1+|u|^{\alpha}}<\infty .
$$

(ii) $C_{1,1}$ denotes the space of all real continuous functionals $\Phi(\cdot)$ on $Y$ such that

$$
\|\Phi\|_{c_{1,1}}=\sup _{u \in Y} \frac{|\Phi(u)|}{1+|u|\|u\|}<\infty .
$$

We further introduce the following:

Definition 4.2. For $C_{\alpha}$ and $C_{1,1}$, we put

$$
L_{\alpha}^{1}=L^{1}\left(0, \infty ; C_{\alpha}\right) \text { and } L_{1,1}^{2}=L^{2}\left(0, \infty ; C_{1,1}\right) .
$$

Moreover, we put, for any $T>0$,

$$
\begin{aligned}
& L_{1,1}^{2, T}=\left\{\Phi(\cdot, \cdot) \in L^{2}\left(0, \infty ; C_{1,1}\right) ; \Phi(t, \cdot)=0 \text { for } t \geqq T\right\} \quad \text { and } \\
& L_{2}^{1, T}=\left\{\Phi(\cdot, \cdot) \in L^{1}\left(0, \infty ; C_{2}\right) ; \Phi(t, \cdot)=0 \text { for } t \geqq T\right\} .
\end{aligned}
$$

Definition 4.3. A family $\{\mu(t, \cdot)\}_{0<t<\infty}$ of positive Borel measures on $X$ will be called basic if it satisfies the conditions (H.1), (H.2) and (H.3) in Definition 2.8, with $\boldsymbol{H}$ replaced by $X$.

The following lemma corresponds to Lemma 1 in Foiaș [5, p. 246].

LEMMA 4.4. Let $\{\mu(t, \cdot)\}_{0<t<\infty}$ be a basic family of Borel measures on $X$. Then, for each $T>0$,

$$
F(\Phi)=\int_{0}^{\infty} \int_{X} \Phi(t, u) d \mu(t, u) d t
$$

makes sense for any $\Phi \in L_{2}^{1} \cup L_{1,1}^{2, T}$. Moreover, we have the following estimates.

$$
\begin{gathered}
\|F\|_{L_{2}^{1^{*}}}=\left\|\int_{X}\left(1+|u|^{2}\right) d \mu(\cdot, u)\right\|_{L^{\infty}(0, \infty)} \text { and } \\
\|F\|_{L_{1,1}^{2, T^{*}}} \leqq\left\|\int_{X}\left(1+|u|^{2}\right) d \mu(\cdot, u)\right\|_{L^{\infty}(0, \infty)}^{1 / 2} \cdot\left\|\int_{X}\left(1+\|u\|^{2}\right) d \mu(\cdot, u)\right\|_{L^{1}(0, T)}^{1 / 2}
\end{gathered}
$$

A slight modification of Lemmas 3 and 4 in Foiaș [5, p. 254 and p. 264] gives:

THEOREM 4.5. Let $\left\{\mu^{(m)}(t, \cdot)\right\}_{0<t<\infty}$ be a sequence of basic families of Borel measures on $X$ such that

(a) $\sup _{m}\left\|\int_{X}\left(1+|u|^{2}\right) d \mu^{(m)}(\cdot, u)\right\|_{L^{\infty}(0, \infty)}=c_{7}<\infty$,

(b) $\sup _{m}\left\|\int_{X}\right\| u\left\|^{2} d \mu^{(m)} d \mu(\cdot, u)\right\|_{L^{1}(0, \infty)}=c_{8}<\infty$.

(c) $\sup _{m}\left\|\mu^{(m)}(\cdot, X)\right\|_{L^{1}(0, T)}=c_{\theta}(T)<\infty$ for each $T<\infty$. 
Let $F^{(m)}$ be a functional defined by

(IRm)

$$
F^{(m)}(\Phi)=\int_{0}^{\infty} \int_{X} \Phi(t, u) d \mu^{(m)}(t, u) d t
$$

Then, we have:

(1) There exists a functional $F, a w^{*}$-cluster point of $\left\{F^{(m)}\right\}$ in $L_{2}^{1^{*}}$.

(2) $F$ is also a $w^{*}$-cluster point of $\left\{F^{(m)}\right\}$ in $L_{1,1}^{2, T^{*}}$ for any $T>0$.

(3) There exists a basic family $\{\mu(t, \cdot)\}_{0<t<\infty}$ such that

$$
F(\Phi)=\int_{0}^{\infty} \int_{X} \Phi(t, u) d \mu(t, u) d t
$$

for all $\Phi \in L_{0}^{1}$.

In addition to the conditions (a)-(c), we assume:

(d) The functional $|\cdot|^{2}$ is uniformly integrable with respect to almost all $\mu^{(m)}(t, \cdot)(0<t<\infty, m=1,2, \cdots)$, that is, for any $\varepsilon>0$, there exists an $0<r_{\varepsilon}<\infty$ such that

$$
\int_{\left\{u \in X ;|u| \geqq r_{\varepsilon}\right\}}|u|^{2} d \mu^{(m)}(t, u) \leqq \varepsilon
$$

for all $m$ and almost all $t$.

Then, the formula (IR) is valid also for every $\Phi \in L_{2}^{1}$.

COROLLARY 4.6. Under the assumptions (a) and (b) in Theorem 4.5, if all measures $\mu^{(m)}(t, \cdot)$ for $0<t<\infty$ are probability measures, then the measures constructed above are also probability measures for almost all $t \in(0, \infty)$.

See Remark in Foiaș [5, p. 263].

REMARK 4.7. To prove Theorem 4.5, we use the property (P.3) of $P_{m}$ which is important to define a desired measure by Daniell's integral.

5. Proof of Theorem A. In order to restate Theorem A more precisely, we need some notation.

We consider the initial boundary value problem of the Navier-Stokes equation, although it is not necessary to solve it.

We define the forms $a$ and $b$ by

$$
a(u, v)=((u, v))=\int_{M} g_{i j} \nabla_{k} u^{i} \nabla^{k} v^{j} d_{g} x
$$

and

$$
b(u, v, w)=\left(\nabla_{u} v, w\right)=\int_{M} g_{i j}\left\{u^{k} \frac{\partial}{\partial x^{k}} v^{i}+\Gamma_{k l}^{i} u^{k} v^{l}\right\} w^{j} d_{g} x
$$

for $u, v, w \in \boldsymbol{X}(M)$. Then, we have: 
LEMma 5.1. (1) The from $b$ can be regarded as a trilinear continuous form on $\boldsymbol{V} \times \boldsymbol{V} \times \boldsymbol{W}$ or $\boldsymbol{H} \times \boldsymbol{V} \times \boldsymbol{V}^{\boldsymbol{s}}$ satisfying

$$
|b(u, v, w)| \leqq\|u\|\|v\|\|w\|_{w} \quad \text { or } \quad|b(u, v, w)| \leqq c_{10}|u|\|v\|\|w\|_{s}
$$

for some $s \geqq d / 2$, where $c_{10}$ is a constant related to the Sobolev imbedding.

(2) For $u \in \boldsymbol{V}$ and $v, w \in \boldsymbol{W}$, we have

$$
b(u, v, w)=-b(u, w, v) .
$$

For the proof, see, for example, Lemmas 1.1-1.3 in Chap. II and Lemma 4.1 in Chap. III of Temam [21].

REMARK 5.2. (a) For $u, v \in V$, we denote by $B(u, v)$ the linear continuous form on $W$ defined by

$$
(B(u, v), w)=b(u, v, w) \text { for } w \in \boldsymbol{W} .
$$

Clearly, $B(u, v)=\nabla_{u} \cdot v$ for $u, v \in X(M)$. By (5.1), we have

$$
\|B(u, v)\|_{W^{\prime}} \leqq\|u\|\|v\| \quad \text { or } \quad\|B(u, v)\|_{-s} \leqq c_{10}|u|\|v\| \text {. }
$$

(b) We also define a linear operator $A$ from $\boldsymbol{V}$ onto $\boldsymbol{V}^{-1}$ by

$$
(A u, v)=a(u, v) \text { for } u, v \in V .
$$

Here, we use $(\cdot, \cdot)$ on the left-hand side also as the duality pairing between $\boldsymbol{V}$ and $\boldsymbol{V}^{-1}$.

Now, we remark that as a corollary to Definition 2.7 in $\S 2$, we have:

Lemma 5.3. (1) Any test functional $\Phi(\cdot, \cdot)$ can be extended to $[0, \infty) \times V^{-s}$ so that

$$
|\Phi(t, u)-\Phi(t, v)| \leqq c_{11}\|u-v\|_{-s}
$$

for all $t \in[0, \infty)$ and $u, v \in \boldsymbol{V}^{-s}$, where $c_{11}=\sup _{t \in \boldsymbol{R}, u \in V}\left\|\Phi_{u}(t, u)\right\|_{s}$. Moreover, this extension is continuous from $[0, \infty) \times \boldsymbol{H}_{\text {weak }}$ to $\boldsymbol{R}$.

(2) Any $\Phi \in \mathrm{TF}$ may be extended as a functional on $[0, \infty) \times \boldsymbol{H}$ and we have

$$
|\Phi(t, u)| \leqq c_{12}+c_{13}|u| \text { for all }(t, u)
$$

with some constants $c_{12}$ and $c_{13}$ depending on $\Phi$.

(3) If a test functional $\Phi(\cdot, \cdot)$ satisfies $\Phi(t, u)=0$ for a certain $t \in[0, \infty)$ and for all $u$ with $\|u\|$ large enough, then actually $\Phi(t, \cdot)=0$.

Proof. See, Foiaș [5, p. 253].

After these preliminaries, we are in a position to restate Theorem A as follows: 
THEOREM $\mathrm{A}^{\prime}$. Let $f(\cdot) \in L^{2}\left(0, \infty ; \boldsymbol{V}^{-1}\right)$ be given and suppose a Borel measure $\mu_{0}$ on $\boldsymbol{H}$ satisfies

$$
\int_{H}\left(1+|u|^{2}\right) d \mu_{0}(u)<\infty .
$$

Then, there exists a basic family $\{\mu(t, \cdot)\}_{0<t<\infty}$ of Borel measures on $\boldsymbol{H}$ such that

$$
\begin{aligned}
& \int_{0}^{\infty} \int_{H} \Phi_{t}(t, u) d \mu(t, u) d t+\int_{H} \Phi(0, u) d \mu_{0}(u) \\
& \quad=\int_{0}^{\infty}\left[\int_{H}\left\{\nu a\left(u, \widetilde{\Phi}_{u}(t, u)\right)+b\left(u, u, \widetilde{\Phi}_{u}(t, u)\right)-\left\langle f(t), \Phi_{u}(t, u)\right\rangle\right\} d \mu(t, u)\right] d t
\end{aligned}
$$

for any $\Phi \in \mathrm{TF}$ with compact support in $t$, i.e., there exists a constant $T$ depending on $\Phi$ such that $\Phi(t, \cdot)=0$ for $t \geqq T$.

Moreover, it satisfies the following energy inequality of strong form.

$$
\begin{aligned}
& \frac{1}{2} \int_{H} \psi\left(|u|^{2}\right) d \mu(t, u)+\nu \int_{0}^{t}\left[\int_{H} \psi^{\prime}\left(|u|^{2}\right)\|u\|^{2} d \mu(\tau, u)\right] d \tau \\
& \quad \leqq \frac{1}{2} \int_{H} \psi\left(|u|^{2}\right) d \mu_{0}(u)+\int_{0}^{t}\left[\int_{H} \psi^{\prime}\left(|u|^{2}\right)(f(\tau), u) d \mu(\tau, u)\right] d \tau
\end{aligned}
$$

for $0<t<\infty$ and $\psi \in C^{1}[0, \infty)$ satisfying

$$
0 \leqq \psi^{\prime}(t) \leqq \sup _{s \in[0, \infty)} \psi^{\prime}(s)<\infty .
$$

The proof of Theorem $\mathrm{A}^{\prime}$ consists of several steps.

Step 1. Using the Galerkin approximation of the Navier-Stokes equation, we may construct a basic family $\left\{\mu^{(m)}(t, \cdot)\right\}_{0<t<\infty}$ for each $m$. Moreover, $\left\{\mu^{(m)}(t, \cdot)\right\}_{0<t<\infty}$ satisfies the conditions (a)-(d) in Theorem 4.5.

So, we have a basic family $\{\mu(t, \cdot)\}_{0<t<\infty}$ of measures as a candidate for the solution of Problem (II).

Step 2. We show that $\{\mu(t, \cdot)\}_{0<t<\infty}$ constructed above satisfies the properties in Theorem $\mathrm{A}^{\prime}$.

Sketch of the Proof of Theorem A'. By standard argument, we can show that there exists an orthonormal basis $\left\{w_{(j)}\right\}$ of $\boldsymbol{H}$, orthogonal in $V$, such that

$$
a\left(w_{(j)}, v\right)=\lambda_{j}\left(w_{(j)}, v\right) \text { for any } v \in \boldsymbol{V},
$$

where $\lambda_{j} \geqq 0$. By a suitable choice of the indices, we may suppose that

$$
0<\lambda_{1} \leqq \lambda_{2} \leqq \cdots \leqq \lambda_{n} \leqq \cdots \rightarrow \infty .
$$

Obviously, $\lambda_{1}$ satisfies 


$$
\frac{1}{\sqrt{\lambda_{1}}}=\sup _{u \in V} \frac{|u|}{\|u\|}
$$

For each $m$, we define the orthogonal projection $P_{m}$ in $\boldsymbol{H}$ by

$$
P_{m} u=\sum_{j=1}^{m}\left(u, w_{(j)}\right) w_{(j)} .
$$

By regarding the scalar product $(\cdot, \cdot)$ in $(5.8)$ as a suitable duality pairing, we can extend the operator $P_{m}$ as an operator from $\boldsymbol{V}^{-s}$ or $\boldsymbol{W}^{\prime}$ to $P_{m} \boldsymbol{H}$. Moreover, $P_{m}$ satisfies the properties (P.1)-(P.3) in $\S 4$ with $X=\boldsymbol{H}, Y=\boldsymbol{V}$ and $D=V^{s+2}$.

From each $m$, putting $u_{(m)}(t)$ as

$$
u_{(m)}(t)=\sum_{j=1}^{m} h_{j m}(t) w_{(j)},
$$

we seek functions $\left\{h_{j m}(t)\right\}$ satisfying

$$
\left(u_{(m)}^{\prime}(t), w_{(j)}\right)+\nu a\left(u_{(m)}(t), w_{(j)}\right)+b\left(u_{(m)}(t), u_{(m)}(t), w_{(j)}\right)=\left(f(t), w_{(j)}\right)
$$

for $t \in(0, \infty)$ and $j=1,2, \cdots, m$, and

$$
u_{(m)}(0)=u_{0 m}=P_{m} u_{0} \text {. }
$$

LEMMA 5.4. There exists a unique solution $u_{(m)}(t) \in C^{1}\left([0, \infty) ; P_{m} \boldsymbol{H}\right)$ of the equation

$$
\frac{d u_{(m)}(t)}{d t}+\nu P_{m} A u_{(m)}(t)+P_{m} B\left(u_{(m)}(t), u_{(m)}(t)\right)=P_{m} f(t) \quad \text { in } P_{m} \boldsymbol{H}
$$

satisfying (5.10). Moreover,

$$
\begin{gathered}
\frac{1}{2} \frac{d}{d t}\left|u_{(m)}(t)\right|^{2}+\nu\left\|u_{(m)}(t)\right\|^{2}=\left(f(t), u_{(m)}(t)\right), \\
\left|u_{(m)}(t)\right|^{2}+\nu \int_{0}^{t}\left\|u_{(m)}(s)\right\|^{2} d s \leqq\left|u_{(m)}(0)\right|^{2}+\frac{1}{\nu} \int_{0}^{\infty}\|f(s)\|_{-1}^{2} d s .
\end{gathered}
$$

REMARK 5.5. The sequence $\left\{u_{(m)}(t)\right\}$ constructed above is called the Galerkin approximation of the Navier-Stokes equation. In fact, from them, we may extract a subsequence converging to a weak solution of the Navier-Stokes equation. As we mentioned before, this fact is not necessary to construct $\{\mu(t, \cdot)\}_{0<t<\infty}$.

Defining an operator $S^{(m)}(t)$ by $S^{(m)}(t) P_{m} u_{0}=u_{(m)}(t)$, we put

$$
\mu^{(m)}(t, \omega)=\mu_{0}^{(m)}\left(\left(S^{(m)}(t)\right)^{-1}\left(\omega \cap P_{m} \boldsymbol{H}\right)\right)
$$

for $t \in(0, \infty)$ and every Borel set $\omega \subset \boldsymbol{H}$, where

$$
\mu_{0}^{(m)}(\omega)=\mu_{0}\left(P_{m}^{-1}\left(\omega \cap P_{m} \boldsymbol{H}\right)\right) \text { for } m=1,2, \cdots .
$$


Then, we may verify that $\left\{\mu^{(m)}(t, \cdot)\right\}_{0<t<\infty}$ is a basic family of measures on $\boldsymbol{H}$, and that the sequence $\left\{\left\{\mu^{(m)}(t, \cdot)\right\}_{0<t<\infty}\right\}_{m=1}^{\infty}$ satisfies the conditions (a), (b), (c) and (d) in Theorem 4.5.

To proceed further, we need the following which corresponds to Lemma 2, in Foiaș [5, p. 249].

Lemma 5.6. Let $\{\mu(t, \cdot)\}_{0<t<\infty}$ be a basic family on $\boldsymbol{H}$. Assume that $f(\cdot) \in L^{2}\left(0, \infty ; V^{-1}\right)$ and $\Phi(\cdot, \cdot) \in \mathrm{TF}$. Then

$$
\begin{aligned}
\varphi(\Phi)=\int_{0}^{\infty}\left[\int _ { H } \left\{-\Phi_{t}(t, u)+\nu a\left(u, \widetilde{\Phi}_{u}(\right.\right.\right. & t, u))+b\left(u, u, \widetilde{\Phi}_{u}(t, u)\right) \\
& \left.\left.-\left\langle f(t), \Phi_{u}(t, u)\right\rangle\right\} d \mu(t, u)\right] d t
\end{aligned}
$$

makes sense. Moreover, defining $\Phi_{m}$ as

$$
\Phi_{m}(t, u)=\Phi\left(t, P_{m} u\right), \text { for all }(t, u) \in[0, \infty) \times \boldsymbol{H},
$$

we get $\Phi_{m}(\cdot, \cdot) \in \mathrm{ETF}$ and

$$
\varphi\left(\Phi_{m}\right) \rightarrow \varphi(\Phi) \text { for } m \rightarrow \infty .
$$

Corollary in Foiaș [5, p. 266] corresponds to:

Lemma 5.7. Let $\{\mu(t, \cdot)\}_{0<t<\infty}$ be a basic family of measures constructed from $\left\{\mu^{(m)}(t, \cdot)\right\}_{0<t<\infty}$ satisfying the assumptions (a)-(d) in Theorem 4.5 . Then, the formula (IR) is valid for all functionals $\Psi_{\Phi}(\cdot, \cdot)$ defined by

$$
\begin{aligned}
\Psi_{\Phi}(t, u)=-\Phi_{t}(t, u)+\nu a\left(u, \widetilde{\Phi}_{u}(t, u)\right)+b\left(u, u, \widetilde{\Phi}_{u}(t, u)\right) & -\left\langle f(t), \Phi_{u}(t, u)\right\rangle,
\end{aligned}
$$

for $\Phi \in \mathrm{ETF}$ with compact support in $t$.

Combining these, we get the first half of Theorem $\mathrm{A}^{\prime}$. The energy inequality of strong form is proved in the same way as Proposition 1 in Foiaș [5, p. 291].

REMARK 5.8. Let $F \in L_{2}^{1 *}$ be the functional attached to $\{\mu(t, \cdot)\}_{0<t<\infty}$. Using the above notation, we see that $\{\mu(t, \cdot)\}_{0<t<\infty}$ is a solution of Problem (II) if and only if

$$
F\left(\Psi_{\oplus}\right)=\int_{H} \Phi(0, u) d \mu_{0}(u)
$$

holds for any $\Phi(\cdot) \in \mathrm{ETF}$ with compact support in $t$. Indeed, $\Psi_{\Phi}$ for such $\Phi$ belongs to $L_{1,1}^{2, T} \cap L_{2}^{1, T}$ which is dense in $L_{2}^{1, T}$.

6. Fourier-Stieltjes transformations of measures and the meaning of the second order functional derivatives.

Definition 6.1. By a Fourier-Stieltjes transform of a bounded Borel 
measure $\mu$ on $\boldsymbol{H}$, we mean the functional $\hat{\mu}$ defined on $\widetilde{\boldsymbol{H}}$ by the formula

$$
\widehat{\mu}(\eta)=\int_{\boldsymbol{H}} e^{i\langle u, \eta\rangle} d \mu(u) \text { for } \eta \in \tilde{\boldsymbol{H}} \text {. }
$$

REMARK 6.2. (1) If $\mu$ is a probability measure on $\boldsymbol{H}$, then $\hat{\mu}$ will be called the characteristic functional of $\mu$. In this case, $\hat{\mu}$ is continuous on $\tilde{\boldsymbol{H}}$ and is positive definite, that is, for any complex numbers $\zeta_{j}$ and any elements $\eta_{j} \in \tilde{\boldsymbol{H}}, j=1,2, \cdots, m$, we have

$$
\sum_{j, k=1}^{m} \zeta_{j} \bar{\zeta}_{k} \hat{\mu}\left(\eta_{j}-\eta_{k}\right) \geqq 0
$$

(2) The correspondence of $\mu$ to $\hat{\mu}$ is one-to-one and onto from Borel probability measures on $\boldsymbol{H}$ \} to continuous positive definite functionals on $\tilde{\boldsymbol{H}}$ with value 1 at 0$\}$. (See, Theorem 1 in Gelfand and Vilenkin [8, p. 348]).

Lemma 6.3. (cf. Foiaș [6, Lemma 1, p. 110]) (1) If $\mu$ is any Borel probability measure on $\boldsymbol{H}$ satisfying

$$
\int_{H}|u|^{2} d \mu(u)<\infty,
$$

then $\hat{\mu}(\cdot)$ is positive definite and differentiable in $\tilde{\boldsymbol{H}}$ and its Fréchet differential $\hat{\mu}_{\eta}(\cdot)$ satisfies

$$
\left\langle\hat{\mu}_{\eta}(\eta), \xi\right\rangle=i \int_{\boldsymbol{H}}\langle u, \xi\rangle e^{i\langle u, \eta\rangle} d \mu(u) \text { for } \xi \in \tilde{\boldsymbol{H}} \text {. }
$$

Moreover, $\hat{\mu}_{\eta \eta}$ exists as an operator from $\tilde{\boldsymbol{H}}$ to $\boldsymbol{H}$ satisfying

$$
\operatorname{trace}_{\tilde{H} \rightarrow \boldsymbol{H}}\left[-\hat{\mu}_{\eta \eta}(0)\right]=\int_{\boldsymbol{H}}|u|^{2} d \mu(u) \text {. }
$$

(2) In addition to (6.1), if we suppose that

$$
\int_{\boldsymbol{H}}\|u\|^{2} d \mu(u)<\infty \text {, }
$$

then $\hat{\mu}_{\eta}(\eta) \in V$ and

$$
\left(\left(\hat{\mu}_{\eta}(\eta), \tilde{\xi}\right)\right)=i \int_{\boldsymbol{H}}((u, \tilde{\xi})) e^{i\langle u, \eta\rangle} d \mu(u) \quad \text { for } \quad \xi \in \widetilde{\boldsymbol{V}} .
$$

Moreover, $\hat{\mu}_{\eta \eta}$ can be regarded as an operator from $\widetilde{\boldsymbol{V}}$ to $\boldsymbol{V}$ satisfying

$$
\operatorname{trace}_{\tilde{V} \rightarrow V}\left[-\hat{\mu}_{\eta \eta}(0)\right]=\int_{\boldsymbol{H}}\|u\|^{2} d \mu(u) \text {. }
$$

(3) Conversely, if $W$ is a positive definite functional on $\widetilde{\boldsymbol{H}}$ such that $W(0)=1, W_{\eta \eta}$ exists and

$$
\operatorname{trace}_{\widetilde{\boldsymbol{H}} \rightarrow \boldsymbol{H}}\left[-W_{\eta \eta}(0)\right]<\infty,
$$


then $W=\hat{\mu}$ for a uniquely determined Borel probability measure $\mu$ on $\boldsymbol{H}$ satisfying (6.1).

Extending the statement appearing at the end of Foias [6], we get:

Proposition 6.4. Let $\mu$ be a probability measure on $\boldsymbol{H}$ such that

$$
\int_{H}\|u\|_{8}^{2} d \mu(u)<\infty
$$

with $s>d / 2$. Then, for any $j, k$ and for any $x, y \in M$, we have,

$$
-\left\langle\hat{\mu}_{\eta \eta}(\eta) \delta_{x} e^{j}, \delta_{y} e^{k}\right\rangle=\int_{H} u^{j}(x) u^{k}(y) e^{i\langle u, \eta\rangle} d \mu(u),
$$

where $\delta_{x} e^{j}$ denotes the functional on $V^{s}$ defined by $\delta_{x} e^{j}(u)=u^{j}(x)$. Moreover, the left-hand side of (6.8) is continuous in $(x, y) \in \bar{M} \times \bar{M}$.

Proof. The equality (9.14) in Foias [5, p. 111] is restated as

$$
\hat{\mu}_{\eta \eta}(\eta) \xi=-\int_{\boldsymbol{H}}\langle u, \xi\rangle u e^{i\langle u, \eta\rangle} d \mu(u) \in \boldsymbol{H}
$$

for all $\eta, \xi \in \widetilde{\boldsymbol{H}}$. Combining (6.9) with (6.7), we have

$$
\left|\left\langle\hat{\mu}_{\eta \eta}(\eta) \xi^{(1)}, \xi^{(2)}\right\rangle\right| \leqq \int_{H}\|u\|_{s}^{2} d \mu(u) \cdot\left\|\xi^{(1)}\right\|_{-s} \cdot\left\|\xi^{(2)}\right\|_{-s}
$$

so that the bilinear functional

$$
\xi^{(1)}, \xi^{(2)} \rightarrow\left\langle\hat{\mu}_{\eta \eta}(\eta) \xi^{(1)}, \xi^{(2)}\right\rangle
$$

extends by continuity to a bilinear continuous functional on the whole $\widetilde{\boldsymbol{V}}^{-s}$. On the other hand, as $s>d / 2, V^{s}$ is continuously imbedded in the set $\boldsymbol{X}^{0}(\boldsymbol{M})$ of continuous vector fields on $M$ by Sobolev's imbedding theorem. So the Dirac functional $\delta_{x} e^{j}$, which sends $u$ to $u^{j}(x)$, is well-defined and continuous on $V^{s}$ for all $x \in \bar{M}$ and $j=1,2, \cdots, d$. By (6.9), we have

$$
\begin{aligned}
\left\langle\hat{\mu}_{\eta \eta}(\eta) \delta_{x} e^{j}, \delta_{y} e^{k}\right\rangle & =-\int_{H}\left\langle u, \delta_{x} e^{j}\right\rangle\left\langle u, \delta_{y} e^{k}\right\rangle e^{i\langle u, \eta\rangle} d \mu(u) \\
& =-\int_{H} \delta_{x}\left(u^{j}\right) \delta_{y}\left(u^{k}\right) e^{i\langle u, \eta\rangle} d \mu(u) .
\end{aligned}
$$

This implies (6.8) and the last assertion follows immediately q.e.d.

On the other hand, if a measure $\mu$ satisfies

$$
\int_{H}\|u\|^{2} d|\mu|(u)<\infty,
$$

where $|\mu|$ denotes the modulus of $\mu$, then for any $v \in V^{s}$, the functional $b(u, u, v)$ in $u$ belongs to $L^{1}(\mu)$. This implies that the integral 


$$
\int_{H} b(u, u, v) e^{i\langle u, \eta\rangle} d \mu(u)
$$

makes sense for all $\eta \in \widetilde{\boldsymbol{H}}$; and is a continuous linear functional in $v$ on $\boldsymbol{V}^{\boldsymbol{s}}$. Therefore it defines an element $-i(L \hat{\mu})(\eta)$ of $\boldsymbol{V}^{-\boldsymbol{s}}$. That is, we have:

Definition 6.5. For any measure $\mu$ on $\boldsymbol{H}$ satisfying (6.13), we define an operator $L$ by

$$
((L \hat{\mu})(\eta), v)=i \int_{H} b(u, u, v) e^{i\langle u, \eta\rangle} d \mu(u)
$$

for all $v \in \boldsymbol{V}^{s}$ and $\eta \in \tilde{\boldsymbol{H}}$.

Define the space $\mathrm{FSM}_{2}$ as the Fourier-Stieltjes image of the space of Borel measures on $\boldsymbol{H}$ satisfying (6.13) with norm

$$
\|\hat{\mu}\|=\int_{\boldsymbol{H}}\|u\|^{2} d|\mu|(u) \text {. }
$$

Then the operator $L$ is bounded from $\mathrm{FSM}_{2}$ to $\boldsymbol{B}\left(\tilde{\boldsymbol{H}}, \boldsymbol{V}^{-8}\right)$. In fact,

$$
\|L \hat{\mu}\|_{B\left(\tilde{\boldsymbol{H}}, V^{-1}\right)}=\sup _{\eta \in \tilde{\boldsymbol{H}},|\eta|=1}\|(L \hat{\mu})(\eta)\|_{-s} \leqq\|\hat{\mu}\| .
$$

To state one of the main results in this section, we need the following lemma which is proved in Ebin and Marsden [4]. We denote by $\boldsymbol{H}_{\sigma, N}^{1}(M)$ the closure of the space $\left\{u \in \boldsymbol{X}_{\sigma}(\bar{M}):\left.u \cdot \boldsymbol{n}\right|_{\partial M}=0\right\}$ in $\boldsymbol{L}^{2}(M)$, and by $\boldsymbol{L}^{2}\left(\mathrm{ST}^{2}(M)\right)$ the closure of the space of sections from $M$ to $\mathrm{ST}^{2}(M)$ in $\left(\boldsymbol{L}^{2}(M)\right)^{n(n-1) / 2}$. Here, $\boldsymbol{n}$ stands for the unit exterior normal on $\partial M$.

Lemma 6.6. Define an operator $T$ by

$$
(T v)_{j k}(x)=\frac{1}{2}\left(\nabla_{k} \widetilde{v}_{j}(x)+\nabla_{j} \widetilde{v}_{k}(x)\right) .
$$

Then $T$ is one-to-one and onto from $\boldsymbol{H}_{o, N}^{1}(M)$ to $\boldsymbol{L}^{2}\left(\mathbf{S T}^{2}(M)\right)$.

THEOREM 6.7. Let $\mu$ be a Borel probability measure on $\boldsymbol{H}$ satisfying (6.7). Then

(i ) $\delta^{2} \hat{\mu}(\eta) / \delta \eta_{i}(x) \delta \eta_{j}(x)$ exists and belongs to $C(\bar{M})$ for each $i, j$.

(ii) For each $v \in V^{s}$,

$$
((L \hat{\mu})(\eta), v)=\left\langle\frac{\delta^{2} \hat{\mu}(\eta)}{\delta \eta_{i}(x) \delta \eta_{j}(x)},(T v)_{j k}(x)\right\rangle
$$

(iii) $\frac{\delta^{2} \hat{\mu}(\eta)}{\delta \eta_{i}(x) \delta \eta_{j}(x)} \frac{\partial}{\partial x^{i}} \otimes \frac{\partial}{\partial x^{j}}$

belongs to $\mathrm{ST}_{2}(M)$ as a continuous element.

Proof. By the kernel theorem of Schwartz [8], (6.11) implies that 
there exist distribution kernels $K(\eta)^{i j}(x, y)$ on $M \times M$ such that for $\xi^{(1)}$, $\xi^{(2)} \in \Lambda_{o}^{1}(M)$,

$$
\left\langle\hat{\mu}_{\eta \eta}(\eta) \xi^{(1)}, \xi^{(2)}\right\rangle=\int_{M \times M} K(\eta)^{i j}(x, y) \xi_{i}^{(1)}(x) \xi_{j}^{(2)}(y) d_{g} x d_{g} y,
$$

where the integral on the right-hand side must be symbolically interpreted. Combining this with (6.8) and the definition of the functional derivative, we have, as distributions,

$$
\frac{\delta^{2} \hat{\mu}(\eta)}{\delta \eta_{i}(x) \delta \eta_{j}(y)}=-\int_{H} u^{i}(x) u^{j}(y) e^{i\langle u, \eta\rangle} d \mu(u)=\left\langle\hat{\mu}_{\eta \eta}(\eta) \delta_{x} e^{j}, \delta_{y} e^{k}\right\rangle .
$$

Since the above holds as continuous functions on $\bar{M} \times \bar{M}$, we get (i).

Using the above definition of $L$ and Proposition 6.4, we get

$$
\begin{aligned}
((L \hat{\mu})(\eta), v) & =i \int_{H} b(u, u, v) e^{i\langle u, \eta\rangle} d \mu(u)=-i \int_{H} b(u, u, v) e^{i\langle u, \eta\rangle} d \eta(u) \\
& =-i \int_{H}\left[\int_{M}\left(\nabla_{k} \widetilde{v}\right)_{j}(x) u^{j}(x) u^{k}(x) d_{g} x\right] e^{i\langle u, \eta\rangle} d \mu(u) \\
& =-i \int_{M}\left(\nabla_{k} \widetilde{v}\right)_{j}(x)\left[\int_{H} \delta_{x}\left(u^{j}\right) \delta_{x}\left(u^{k}\right) e^{i\langle u, \eta\rangle} d \mu(u)\right] d_{g} x .
\end{aligned}
$$

where we have used (6.7) and Fubini's theorem. So, we have

$$
((L \hat{\mu})(\eta), v)=i \int_{M}\left(\nabla_{k} \widetilde{v}\right)_{j}(x)\left[\hat{\mu}_{\eta \eta}(\eta)\left(\delta_{x} e^{j}, \delta_{x} e^{k}\right)\right] d_{g} x
$$

for all $\eta \in \tilde{\boldsymbol{H}}$ and $v=v^{j} \partial / \partial x^{j} \in \boldsymbol{V}$.

Because (6.19) holds with the term $\left(\nabla_{k} \widetilde{v}\right)_{j}(x)$ replaced by $(T v)_{j k}(x)$, we have an element in $\operatorname{ST}^{2}(M)$ for $v \in V^{s}$ by Lemma 6.6. This implies that

$$
\frac{\delta^{2} \hat{\mu}(\eta)}{\delta \eta_{i}(x) \delta \eta_{j}(x)} \frac{\partial}{\partial x^{i}} \otimes \frac{\partial}{\partial x^{j}}
$$

belongs to $\mathrm{ST}_{2}(M)$ as a continuous element. This leads us to (ii) and (iii).

q.e.d.

Now, we extend the above theorem to the following:

COROLlary 6.8. Let $\mu$ be a Borel probability measure on $\boldsymbol{H}$ satisfying (6.13). Then, there exists a distribution $K(\eta)^{i j}(x) \partial / \partial x^{i} \otimes \partial / \partial x^{j}$ in $\mathrm{ST}_{2}(M)$ such that

$$
((L \hat{\mu})(\eta), v)=i\left\langle K(\eta)^{j k}(x),(T v)_{j k}(x)\right\rangle
$$

for $v \in \stackrel{\circ}{\boldsymbol{X}}_{\sigma}(M)$.

Proof. Using the third equality in (6.18), which is valid when $\mu$ satisfies (6.13), we have 


$$
((L \hat{\mu})(\eta), v)=-i \int_{H}\left[\int_{M}(T v)_{j k}(x) u^{j}(x) u^{k}(x) d_{g} x\right] e^{i\langle u, \eta\rangle} d \mu(u) .
$$

This implies, by (5.1),

$$
|((L \hat{\mu})(\eta), v)| \leqq\|T v\|_{s-1}\left(\int_{H}|u|\|u\| d|\mu|(u)\right) .
$$

So there exists a distributional element in $\mathrm{ST}_{2}(M)$ denoted by $K(\eta)^{i j}(x) \partial / \partial x^{i} \otimes$ $\partial / \partial x^{j}$ satisfying $(6.20)$.

REMARK 6.9. By Theorem 6.7, it seems natural to put

$$
\frac{\delta^{2} \hat{\mu}(\eta)}{\delta \eta_{i}(x) \delta \eta_{j}(x)} \frac{\partial}{\partial x^{i}} \otimes \frac{\partial}{\partial x^{j}}=K(\eta)^{k l}(x) \frac{\partial}{\partial x^{k}} \otimes \frac{\partial}{\partial x^{l}} .
$$

Because a measure $\mu$ satisfying (6.13) on $\boldsymbol{H}$ may be approximated weakly by a measure $\mu_{\varepsilon}$ satisfying (6.7) on $\boldsymbol{H}$, we may have

$$
\begin{aligned}
((L \hat{\mu})(\eta), & v)=i\left\langle K(\eta)^{i j},(T v)_{i j}\right\rangle \\
= & \lim _{\varepsilon \rightarrow 0}-i \int_{H}\left[\int_{M}(T v)_{j k}(x) u^{j}(x) u^{k}(x) d_{g} x\right] e^{i\langle u, \eta\rangle} d \mu_{\varepsilon}(u) \\
= & \lim _{\varepsilon \rightarrow 0}-i \int_{M}(T v)_{j k}(x)\left[\int_{H} u^{j}(x) u^{k}(x) e^{i\langle u, \eta\rangle} d \mu_{\varepsilon}(u)\right] d_{g} x \\
= & \lim _{\varepsilon \rightarrow 0} i\left\langle\frac{\delta^{2} \widehat{\mu}_{\varepsilon}(\eta)}{\delta \eta_{i}(x) \delta \eta_{j}(x)},(T v)_{i j}(x)\right\rangle=i\left\langle\frac{\delta^{2} \hat{\mu}(\eta)}{\delta \eta_{i}(x) \delta \eta_{j}(x)},(T v)_{i j}(x)\right\rangle .
\end{aligned}
$$

We rather define

$$
\frac{\delta^{2} \hat{\mu}(\eta)}{\delta \eta_{i}(x) \delta \eta_{j}(x)} \frac{\partial}{\partial x^{i}} \otimes \frac{\partial}{\partial x^{j}}=\lim _{\varepsilon \rightarrow 0} \frac{\delta^{2} \hat{\mu}_{\varepsilon}(\eta)}{\delta \eta_{i}(x) \delta \eta_{j}(x)} \frac{\partial}{\partial x^{i}} \otimes \frac{\partial}{\partial x^{j}},
$$

where the limit is taken in $\mathrm{ST}_{2}(M)$ in the sense of distribution.

Finally, we recall the following lemma in Foias [6, p. 115].

LEMMA 6.10. Let $\mu$ be a Borel probability measure on $\boldsymbol{H}$. Then, $\hat{\mu}$ is of $\widetilde{\boldsymbol{V}}^{-s}$ exponential type if and only if $\mu$ is with bounded support in $V^{s}$.

7. Proof of Theorem B. Theorem 1 in Foias [6, p. 106], is restated as follows:

TheOREM 7.1. Let $\{\mu(t, \cdot)\}_{0<t<\infty}$ be a solution of Problem (II) with initial data $\mu_{0}$ satisfying (5.5). Let $W(t, \eta)$ denote the characteristic functional of $\mu(t, \cdot)$ and let $W_{0}(\eta)$ be that of $\mu_{0}$. Then, we have:

(1) $W(t, \eta)$ is defined on $[0, \infty) \times \tilde{\boldsymbol{H}}$ and belongs to $L^{1}(0, \infty)$ with respect to $t$ for each $\eta \in \widetilde{\boldsymbol{H}}$.

(2) For any $\eta \in \widetilde{\boldsymbol{V}}^{s}$ and any $\rho(\cdot) \in C_{0}^{1}[0, \infty)$, we have 


$$
\begin{gathered}
-\int_{0}^{\infty} \rho^{\prime}(t) W(t, \eta) d t+\int_{0}^{\infty} \rho(t)\left[\nu a\left(W_{\eta}(t, \eta), \tilde{\eta}\right)+((L W(t, \cdot))(\eta),(\tilde{\eta})] d t\right. \\
=\rho(0) W_{0}(\eta)+i \int_{0}^{\infty} \rho(t)\langle f(t), \eta\rangle W(t, \eta) d t .
\end{gathered}
$$

Analogously, Theorem 2 in Foias [6, p. 109] is restated as follows:

THEOREM 7.2. Let $W_{0}(\cdot)$ be a positive definite functionl on $\boldsymbol{H}$ such that $W_{0}(0)=1$ and that $W_{0 \eta \eta}(\eta)$ exists with

$$
\operatorname{trace}_{\tilde{H} \rightarrow H}\left[-W_{0 \eta \eta}(0)\right]<\infty .
$$

Then, there exists a solution $W(t, \cdot)$ on $(0, \infty)$ of $(7.1)$ with initial data $W_{0}(\cdot)$, such that, for any $t \in(0, \infty), W(t, \cdot)$ is a positive definite functional on $\widetilde{\boldsymbol{H}}, W(t, 0)=1, W_{\eta \eta}(t, \cdot)$ exist and satisfies

$$
\begin{gathered}
\operatorname{trace}_{\tilde{H} \rightarrow H}\left[-W_{\eta \eta}(\cdot, 0)\right] \in L^{1}(0, \infty) \text { and } \\
\quad \operatorname{trace}_{\tilde{V} \rightarrow \gamma}\left[-W_{\eta \eta}(\cdot, 0)\right] \in L^{\infty}(0, \infty) .
\end{gathered}
$$

The following lemma is elementary.

Lemma 7.3. Let $\varphi_{0}$ be a real number. Let $\varphi(t)$ belong to $L^{\infty}(0, \infty)$. If there exists a function $\psi(t) \in L_{100}^{1}[0, \infty)$ such that

$$
-\int_{0}^{\infty} \rho^{\prime}(t) \varphi(t) d t-\rho(0) \varphi_{0}=\int_{0}^{\infty} \rho(t) \psi(t) d t
$$

for any $\rho(t) \in C_{0}^{1}[0, \infty)$, then (a) $\phi^{\prime}=\psi$ in $D^{\prime}(0, \infty)$, (b) we can make $\varphi$ continuous on $[0, \infty)$ after modifying the values on the set of measure 0 and (c) $\varphi(t)$ converges to $\varphi_{0}$ as $t \rightarrow 0$.

Proof of Theorem $B$. We can derive the following equation from (7.1) by taking $\rho(\cdot) \in C_{0}^{1}(0, \infty)$.

$$
W_{t}(t, \eta)=\nu a\left(W_{\eta}(t, \eta), \tilde{\eta}\right)+((L W(t, \cdot))(\eta), \tilde{\eta})-i\langle f(t), \eta\rangle W(t, \eta),
$$

which holds as distributions on $(0, \infty)$ for each $\eta \in \widetilde{D}$.

As $W(t, \eta)=\hat{\mu}(t, \eta)$, we have readily

$$
\left\langle W_{\eta}(t, \eta), d \varphi\right\rangle=0 \text { for } \varphi \in C_{0}^{\infty}(M),
$$

and

$$
W(t, 0)=1 \text {. }
$$

To prove

$$
\lim _{t \rightarrow 0} W(t, \eta)=W_{0}(\eta)
$$

we must check the conditions in Lemma 7.3. 
For each $\eta \in \widetilde{V}^{s}$, putting $\varphi(t)=W(t, \eta)$, we have already proved that $\varphi(t) \in L^{\infty}(0, \infty)$ and $\varphi(t)$ satisfies the equation in Lemma 7.6 with

$$
\psi(t)=\nu a\left(W_{\eta}(t, \eta), \tilde{\eta}\right)+((L W(t, \cdot))(\eta), \tilde{\eta})-i\langle f(t), \eta\rangle W(t, \eta) .
$$

As $\psi(t)$ belongs to $L_{1 \mathrm{oc}}^{1}[0, \infty)$ because of

$$
\left|\frac{\partial}{\partial t} W(t, \eta)\right| \leqq \int_{H}\left[\nu|\eta|\|u\|_{2}+c_{10}\|u\|^{2}\|\eta\|_{l}+|f(t)||\eta|\right] d \mu(t, u),
$$

we have (7.8). Lastly using (EIS), we have

$$
\int_{H}\|u\|^{2} d \mu(t, u)<\infty \text { for almost every } t
$$

so by Corollary $6.8,((L W(t, \cdot))(\eta), \tilde{\eta})$ may be expressed as

$$
((L W(t, \cdot))(\eta), \tilde{\eta})=\left\langle(T \tilde{\eta})_{j k}(x), \frac{\delta^{2} W(t, \eta)}{\delta \eta_{j}(x) \delta \eta_{k}(x)}\right\rangle
$$

for $\eta \in \tilde{V}^{s}$.

q.e.d.

REMARK 7.4. The above proof of Theorem B indicates that the solution in $\S 5$ also gives a strong solution in our case. Moreover, it is clear from the arguments in $\S 3$ and $\S 6$ that a strong solution yields a solution of Problem (II). As the condition (7.2) seems necessary to define a strong solution by our reasoning, it is natural to ask whether there exists a family of measures satisfying (5.6) without the condition (5.5). If we want to remove the condition trace $\widetilde{H} \rightarrow \boldsymbol{H}_{[}\left[-W_{0 \eta \eta}(0)\right]<\infty$ in Theorem A, we must introduce new notion of solution of (I), which will be named a weak solution. This point will be studied in the forthcomming paper.

8. Proof of Theorem C. To prove Theorem C, we repeat the procedure in $§ 5-6$ with some modifications.

First of all, instead of (5.13), we use

$$
\left|u_{(m)}(t)\right|^{2}+2 \nu \int_{0}^{t}\left\|u_{(m)}(s)\right\|^{2} d s \leqq 2\left|u_{0}\right|^{2}+3\left[\int_{0}^{t}|f(s)| d s\right]^{2}
$$

which is given, for example, in Ladyzhenskaya [17, p. 147]. Then, defining $\left\{\mu^{(m)}(t, \cdot)\right\}_{0<t<\infty}$ as in (5.14), we get

$$
\begin{aligned}
& \int_{\boldsymbol{H}}\left(1+|u|^{2}\right) d \mu^{(m)}(t, u)+2 \nu \int_{0}^{\infty}\left[\int_{\boldsymbol{H}}\|u\|^{2} d \mu^{(m)}(t, u)\right] d t \\
& \quad \leqq \int_{\boldsymbol{H}}\left(1+2|u|^{2}\right) d \mu_{0}(u)+c_{14} \cdot \mu_{0}(\boldsymbol{H})=2 \alpha_{0}+c_{14} \cdot \mu_{0}(\boldsymbol{H})=c_{15} .
\end{aligned}
$$

Here, $c_{14}=3\left[\int_{0}^{\infty}|f(s)| d s\right]^{2}$. 
Secondly, we want to have a constant independent of $m$ and $t \in\left(0, T^{*}\right)$ such that

$$
\int_{H}\|u\|_{l}^{2} d \mu^{(m)}(t, u) \leqq c_{16}<\infty,
$$

where $l$ is the largest integer not exceeding $(d / 2)+1$.

To this end, we begin with the following:

Lemma 8.1. For $w_{(j)}$ introduced in $\S 5$ we have:

(1) $w_{(j)}$ belongs to $\boldsymbol{H}^{l+2}$.

(2) $P_{m}$ satisfies

$$
\left\|P_{m} u\right\|_{l} \leqq\left\|P_{m+1} u\right\|_{l} \leqq\|u\|_{l} .
$$

PRoof. (1) follows from the regularity theorem of a weak solution of elliptic systems. (2) follows if we redefine the norm $\|\cdot\|_{l}$ by

$$
\|u\|_{l}^{2}=\sum_{j=1}^{l}\left|(-\Delta)^{j / 2}\right|^{2}
$$

in view of the property of $w_{(j)}$ and $\lambda_{j}$ in (EVP).

q.e.d.

LEMMA 8.2. As $\partial M=\varnothing$, we have $\boldsymbol{H}^{l}=V^{l}$ and

$$
\|B(u, v)\|_{l} \leqq c_{17} \cdot\|u\|_{l}\|v\|_{l+1}
$$

for $u \in V^{\imath}, v \in V^{l+1}$. Moreover,

$$
\left|(B(u, v), v)_{l}\right| \leqq c_{18} \cdot\|u\|_{l}\|v\|_{l+1}^{2} .
$$

For the proof, see Kato [16].

Lemma 8.3. Let $u_{(m)}(t) \in C^{1}\left([0, \infty) ; P_{m} \boldsymbol{H}\right)$ be a unique solution obtained previously in $\S 5$. For any $\alpha>1$, there exists $T^{*}$ depending on $\alpha,\|f(t)\|_{l}$ and $\left\|u_{0}\right\|_{l}$ such that

$$
\left\|u_{(m)}(t)\right\|_{l} \leqq \alpha\left\|u_{0}\right\|_{l} \quad \text { on } \quad\left[0, T^{*}\right) .
$$

Proof. Using the arguments in Temam [20] and Kato [16], (here, $\partial M=\varnothing$ is used), we have

$$
\frac{1}{2} \frac{d}{d t}\left\|u_{(m)}(t)\right\|_{l}^{2}+\nu\left\|u_{(m)}(t)\right\|_{l+1}^{2} \leqq c_{10} \cdot\left\|u_{(m)}\right\|_{l}^{3}+c_{20} \cdot\|f(t)\|_{l}\left\|u_{(m)}\right\|_{l} .
$$

Comparing the solution $u_{(m)}(t)$ of (5.11) with the solution $y(t)$ of the ordinary differential equation

$$
\frac{d}{d t} y(t)=c_{10} \cdot y(t)^{2}+c_{20} \cdot\|f(t)\|_{l}
$$

with initial data $y(0)=\left\|u_{0}\right\|_{l}$, we have 


$$
\left\|u_{(m)}\right\|_{l} \leqq y(t) \leqq \alpha y(0)
$$

on some interval $\left[0, T^{*}\right)$, where $T^{*}$ is defined by and depends on $y(0)$, $f(\cdot, t)$ and arbitrarily given number $\alpha>1$. For example, $T^{*}=\alpha-1 / \alpha c_{20} y(0)$ when $f(t) \equiv 0$.

q.e.d.

As the functional $W_{0}(\eta)$ is of $\tilde{V}^{-l}$ exponential type, we may take the above $T^{*}$ strictly positive because of the last remark of the above proof. Moreover, from the conditions

$$
\operatorname{trace}_{\tilde{H} \rightarrow H}\left[-W_{0 \eta \eta}\right]<\infty \text { and } \operatorname{trace}_{\tilde{\nu} l \rightarrow V}\left[-W_{0 \eta \eta}\right]<\infty,
$$

and remembering Lemma 6.3 , we have a Borel probability measure $\mu_{0}$ on $\boldsymbol{H}$ satisfying

$$
\int_{H}|u|^{2} d \mu_{0}(u)<\infty \text { and } \int_{H}\|u\|_{l}^{2} d \mu_{0}(u)<\infty .
$$

Therefore, applying the results in $\S 4$ to the case where $X=\boldsymbol{H}, Y=V^{l}$ and $D=V^{l+2}$, we may define a Borel measure $\mu^{(m)}(t, \cdot)$ satisfying the desired inequality on $\left[0, T^{*}\right)$ with $c_{18}=\alpha^{2} \int_{H}\|u\|_{l}^{2} d \mu_{0}(u)<\infty$. This implies that $\left\{\mu^{(m)}(t, \cdot)\right\}_{0<t<\infty}$ satisfies the properties (a)-(d) in Theorem 4.5. So we may apply Proposition 6.4 and (6.19). Combining this with Theorem 7.1 and 7.2, and then with Lemma 7.3, we finished the proof of existence.

9. Concluding remarks. Our aim to write this paper was as follows:

(1) To understand clearly the procedure presented in Foiaș [5], [6] from the point of view of solving F.D.E.

(2) To give an exact meaning to

$$
\frac{\delta^{2} W(t, \eta)}{\delta \eta_{j}(x) \delta \eta_{k}(x)}
$$

which is not given in the above works.

(3) To find another construction of weak, strong or classical solutions of F.D.E. of second order. In the case of first order, we have examples of solutions without classical correspondence. See, Inoue [12], [14].

(4) In connection with (3), we want to find the change of variables formula for F.D.E., which we find for the problem of quantization in Inoue and Maeda [15].

To explain the point (4) above more precisely, let us consider an example. Berger's equation is given by

$$
u_{t}+u u_{x}-\nu u_{x x}=0 \text { for }(x, t) \in \boldsymbol{R} \times(0, \infty) .
$$

It is well-known that this equation is linearized by the Hopf-Cole trans- 
formation, given by $u(x, t)=-2 \nu \partial / \partial x \log v(x, t)$. That is, if $u(x, t)$ satisfies (8.1), then $v(x, t)$ satisfies

$$
v_{t}-\nu v_{x x}=0
$$

and vice versa.

Now, we consider the functor from the Navier-Stokes equation to the Hopf equation in this case, that is, we consider the following F.D.E.'s.

$$
\frac{\partial}{\partial t} U(t, \eta)=\int_{R} \eta(x)\left[i \frac{\partial}{\partial x} \frac{\delta^{2} U(t, \eta)}{\delta \eta(x)^{2}}+\nu \frac{\partial^{2}}{\partial x^{2}} \frac{\delta U(t, \eta)}{\delta \eta(x)}\right] d x
$$

and

$$
\frac{\partial}{\partial t} V(t, \xi)=\int_{R} \nu \xi(x) \frac{\partial^{2}}{\partial x^{2}} \frac{\delta V(t, \xi)}{\delta \xi(x)} d x .
$$

If these functors commute with the Hopf-Cole transformation, then we could construct a solution $U$ of F.D.E. of second order from a solution $V$ of F.D.E. of first order.

Concerning the above problems, we have some affirmative answers to (1) and (2). But most important ones (3) and (4) are left unanswered.

\section{REFERENCES}

[1] A. A. Arsen'ev, Construction of turbulence measure for the system of Navier-Stokes equations, Math. USSR-Sb. 30 (1976), 179-186.

[2] A. A. ARSEN'Ev, On statistical solutions of the Navier-Stokes system of equations, Math. USSR-Sb. 38 (1981), 31-45.

[3] M. D. Donskar and J.L. Lions, Fréchet-Volterra variational equations, boundary value problems and function space integrals, Acta Math. 108 (1962), 147-228.

[4] D. G. Ebin AND J. MARSDEN, Groups of diffeomorphisms and the motion of an incompressible fluid, Ann. of Math. 92 (1970), 102-163.

[5] C. Foisș, Statistical study of Navier-Stokes equations I, Rend. Sem. Mat. Univ. Padova 48 (1973), 219-349.

[6] C. Foraș, Statistical study of Navier-Stokes equations II, Rend. Sem. Mat. Univ. Padova 49 (1973), 9-123.

[7] I. M. Gelfand, Some aspects of functional analysis and algebra, Internat. Congress of Math. in Amsterdam, 1954, North-Holland, Amsterdam, 1957, 253-276.

[8] I. M. Gelfand and N. Ya. Vilenkin, Generalized Functions-Application of Harmonic Analysis, Vol. 4, Academic Press, New York, 1964.

[9] I. I. Gihman and A. V. Skorohod, The Theory of Stochastic Processes I, Springer-Verlag, Berlin, Heidelberg, New York, 1974.

[10] E. HOPF, Statistical hydrodynamics and functional calculus, J. Rational Mech. Anal. 1 (1952), 87-123.

[11] E. HOPF AND E.W. TITT, On certain special solution of the $\Phi$-equation of statistical hydrodynamics, J. Rational Mech. Anal. 2 (1953), 587-591.

[12] A. INOUE, An explicit solution of a certain Schwinger-Dyson equation, Proc. Japan Acad. Ser A. 57 (1981), 477-480. 
[13] A. INoue, On weak, strong and classical solutions of the Hopf equation -an example of F.D.E. of second order, Proc. Japan Acad. Ser. A 62 (1986), 54-57.

[14] A. INoue, Some examples exhibiting the procedures of renormalization and gauge fixing -Schwinger-Dyson equations of first order, Kodai Math. J. 9 (1986), 134-160.

[15] A. INOUE AND Y. MAEDA, On integral transformations associated with a certain Lagrangian -as a prototype of quantization, J. Math. Soc. Japan 37 (1985), 219-244.

[16] T. Kato, Nonstationary flows of viscous and ideal fluids in $\boldsymbol{R}^{3}$, J. Func. Anal. 9 (1972), 296-305.

[17] O.A. Ladyzhenskaya, Mathematical Theory of Viscous Incompressible Flows, 2nd ed., Gordon and Breach, New York, 1963.

[18] O.A. Ladyzhenskaya and A. M. Vershik, Sur l'évolution des mésures détérminées par les équations de Navier-Stokes et la résolution du problème de Cauchy pour l'équation statique de E. Hopf, Annali Scuola Norm. Sup. Pisa 42 (1977), 209-230.

[19] P. Levy, Problèmes Concréts d'Analyse Fonctionelle, Gauthier-Villars, Paris, 1951.

[20] R. Temam, On the Euler equations of incompressible perfect fluids, J. Func. Anal. 20 (1975), 32-43.

[21] R. Temam, Navier-Stokes Equations. Theory and Numerical Analysis, North-Holland, Amsterdam, 1979.

[22] M. I. Vishik AND A.I. KomeCh, On the solvability of the Cauchy problem for the Hopf equation corresponding to a nonlinear hyperbolic equation, Amer. Math. Soc. Transl. (2) 118 (1982), 161-184.

[23] M. I. Vishik, A. I. Koмech and A. V. Fursikov, Some mathematical problems of statistical hydromechanics, Russian Math. Surveys 34 (1979), 149-234.

[24] V. Volterra, Leçons sur les Equations de Lignes, Gauthier-Villars, Paris, 1913.

Department of Mathematics

TOKYO INSTITUTE OF TEChNOLOGY

OH-Okayama, Meguro-Ku

TOKYO, 152

JAPAN 